\title{
Completely order bounded maps on non-commutative $L_{p}$-spaces
}

\section{Erwin Neuhardt ${ }^{1}$}

Received: 14 October 2020/Accepted: 30 April 2021 / Published online: 8 June 2021

(C) The Author(s) 2021

\begin{abstract}
We define norms on $L_{p}(\mathcal{M}) \otimes M_{n}$ where $\mathcal{M}$ is a von Neumann algebra and $M_{n}$ is the space of complex $n \times n$ matrices. We show that a linear map $T: L_{p}(\mathcal{M}) \rightarrow$ $L_{q}(\mathcal{N})$ is decomposable if $\mathcal{N}$ is an injective von Neumann algebra, the maps $T \otimes$ $I d_{M_{n}}$ have a common upper bound with respect to our defined norms, and $p=\infty$ or $q=1$. For $2 p<q<\infty$ we give an example of a map $T$ with uniformly bounded maps $T \otimes I d_{M_{n}}$ which is not decomposable.
\end{abstract}

Keywords Non-commutative $L_{p}$-space $\cdot$ Matrix norm $\cdot$ Completely positive map · Decomposable map

Mathematics Subject Classification 46L07 · 47L25

\section{Introduction}

Completely positive maps on von Neumann algebras have been studied extensively and there are some nice results on such maps (see e.g. [12], Ch. IV.3 and [10], Ch. 11). When combining the order structure with the vector space structure, it is natural to investigate the linear span of completely positive maps. These are called decomposable maps in [4], §1. The decomposable maps from a von Neumann algebra $\mathcal{M}$ into an injective von Neumann algebra $\mathcal{N}$ are the completely bounded maps. This has been shown at about the same time by Haagerup [4], Paulsen [8],

Communicated by Uwe Franz.

Erwin Neuhardt

e.neuhardt@hs-sm.de

1 Faculty of Computer Science, Hochschule Schmalkalden, Blechhammer, 98574 Schmalkalden, Germany 
and Wittstock [14]. Completely bounded maps use the operator norm on matrices of elements of a von Neumann algebra.

For a von Neumann algebra $\mathcal{M}$, the non-commutative $L_{p}$-space $L_{p}(\mathcal{M})$, $1 \leq p<\infty$, can be realized as (in general) unbounded operators on a Hilbert space. Therefore the $L_{p}$-space itself as well as matrices with entries of a $L_{p}$-space have a natural order given by positive operators. This allows us to define completely positive maps and decomposable maps from one $L_{p}$-space into another one. Then there should be a description of decomposable maps using some norm conditions on matrices of $L_{p}$-spaces.

This question has been partially answered by Pisier [9] for linear maps from the Schatten classes $S_{p}$ to $S_{p}$, and Arhancet and Kriegler [1] for linear maps from $L_{p}(\mathcal{M})$ to $L_{p}(\mathcal{N})$, where $\mathcal{M}$ and $\mathcal{N}$ are semifinite, approximately finite dimensional von Neumann algebras. Junge and Ruan [6] give a description of the decomposable norm for finite rank maps from $L_{p}(\mathcal{M})$ to $L_{p}(\mathcal{N})$, where $\mathcal{M}$ and $\mathcal{N}$ are arbitrary von Neumann algebras. All cited articles require the same $p$-index for domain and range space.

Our idea is to derive a norm on matrices of $L_{p}$-spaces from the order structure. This norm is quite similar to the norm used in [1] and [9] (see [9] equation (1.5)). Using this norm, we can characterize decomposable maps from $\mathcal{M}$ to $L_{q}(\mathcal{N})$ where $1 \leq q \leq \infty$ and from $L_{p}(\mathcal{M})$ to $L_{1}(\mathcal{N})$ where $1 \leq p \leq \infty$. In both cases $\mathcal{N}$ must be injective. Then we give an example of von Neumann algebras $\mathcal{M}$ and $\mathcal{N}$ and a linear map from $L_{p}(\mathcal{M})$ to $L_{q}(\mathcal{N})$ where $1 \leq p, q<\infty, q>2 p$ which is completely order bounded with respect to our matricial norms but not decomposable. Therefore this norm cannot characterize decomposable maps for all combinations of $p$ and $q$.

The structure of the article is as follows: In Sect. 2, we give a short description of non-commutative $L_{p}$-spaces in the Haagerup-Terp construction. We also show some properties of matrices of operators. In Sect. 3, we define our norm on matrices and derive some properties of this norm. In Sect. 4, we define completely order bounded maps and show that they are decomposable for some combinations of $p$ and $q$.

\section{Non-commutative $L_{p}$-spaces}

Short descriptions of non-commutative $L_{p}$-spaces in the Haagerup-Terp construction can be found in several publications, but the main source is still [13]. Here, we cite some basic facts from [13] we will use. Let $\mathcal{M}$ be a von Neumann algebra acting on a Hilbert space $\mathcal{H}$, and let $\varphi$ be a normal faithful semifinite weight on $\mathcal{M}$ with modular automorphism group $\sigma_{t}^{\varphi}$. Then the crossed product $\mathcal{M} \rtimes_{\sigma^{\varphi}} \mathbb{R}$ acts on the Hilbert space $L_{2}(\mathbb{R}, \mathcal{H})$ and is the von Neumann algebra generated by the operators $\pi(x)$ and $\lambda(s)$ where

$$
(\pi(x) \xi)(t)=\sigma_{-t}^{\varphi}(x) \xi(t), \quad x \in \mathcal{M}, t \in \mathbb{R}, \xi \in L_{2}(\mathbb{R}, \mathcal{H})
$$

and 


$$
(\lambda(s) \xi)(t)=\xi(t-s), \quad s, t \in \mathbb{R}, \xi \in L_{2}(\mathbb{R}, \mathcal{H}) .
$$

For $s \in \mathbb{R}$ let $W(s)$ be the unitary operator on $L_{2}(\mathbb{R}, \mathcal{H})$ which is defined by

$$
(W(s) \xi)(t)=e^{-\mathrm{ist}} \xi(t), \quad s, t \in \mathbb{R}, \xi \in L_{2}(\mathbb{R}, \mathcal{H}) .
$$

The dual action $\theta$ is then defined by

$$
\theta_{s}(x)=W(s) x W(s)^{*}, \quad x \in \mathcal{M} \rtimes_{\sigma^{\varphi}} \mathbb{R}, s \in \mathbb{R} .
$$

The elements of $\mathcal{M}$ are the fixed points under $\theta$ when $\mathcal{M}$ is identified with $\pi(\mathcal{M})$ :

$$
\pi(\mathcal{M})=\left\{x \in \mathcal{M} \rtimes_{\sigma^{\varphi}} \mathbb{R} \mid \theta_{s}(x)=x \text { for all } s \in \mathbb{R}\right\} .
$$

The crossed product $\mathcal{M} \rtimes_{\sigma^{\varphi}} \mathbb{R}$ has a unique normal faithful semifinite trace $\tau$ which satisfies

$$
\tau\left(\theta_{s}(x)\right)=e^{-s} \tau(x) \text { for all } x \in\left(\mathcal{M} \rtimes_{\sigma^{\varphi}} \mathbb{R}\right)_{+}, s \in \mathbb{R} .
$$

The existence of the trace $\tau$ allows to consider the $\tau$-measurable operators. These are all closed densely defined operators $a$ affiliated with $\mathcal{M} \rtimes_{\sigma^{\varphi}} \mathbb{R}$ which satisfy: For every $\varepsilon>0$ there exists a projection $p \in \mathcal{M} \rtimes_{\sigma^{\varphi}} \mathbb{R}$ such that $p L_{2}(\mathbb{R}, \mathcal{H}) \subseteq \mathcal{D}(a)$ and $\tau(1-p) \leq \varepsilon$. A subspace $\mathcal{D}$ of $L_{2}(\mathbb{R}, \mathcal{H})$ is called $\tau$-dense if for every $\varepsilon>0$ there is a projection $p \in \mathcal{M} \rtimes_{\sigma^{\varphi}} \mathbb{R}$ such that $p L_{2}(\mathbb{R}, \mathcal{H}) \subseteq \mathcal{D}$ and $\tau(1-p) \leq \varepsilon$. Thus the $\tau$ measurable operators are those which are affiliated with $\mathcal{M} \rtimes_{\sigma^{\varphi}} \mathbb{R}$ and have a $\tau$ dense domain. The closure of a $\tau$-measurable operator restricted to a $\tau$-dense subspace is unique. Therefore, for proving some property of a $\tau$-measurable operator it suffices to prove the property on a $\tau$-dense subspace which is contained in the domain of the operator. The $\tau$-measurable operators form a topological $*$-algebra. When two $\tau$-measurable operators are added or multiplied, we have to create the closure of the sum or the product which always exist and are unique. More details for $\tau$-measurable operators can be found in [13], Chapter I or [12], Chapter IX.2.

The action $\theta$ can be extended to all $\tau$-measurable operators. The space $L_{p}(\mathcal{M})$, $1 \leq p \leq \infty$, consists of all $\tau$-measurable operators $a$ for which

$$
\theta_{s}(a)=e^{-\frac{s}{p}}(a) \text { for all } s \in \mathbb{R} .
$$

There is a one-to-one correspondence between the elements of the predual $\mathcal{M}_{*}$ and the elements of $L_{1}(\mathcal{M})$. This correspondence defines the linear functional $t r$ : $L_{1}(\mathcal{M}) \rightarrow \mathbb{C}$ by $\operatorname{tr}\left(h_{\varphi}\right)=\varphi(1)$, where $h_{\varphi} \in L_{1}(\mathcal{M})$ is the operator assigned to $\varphi \in \mathcal{M}_{*}$. This linear functional has the following properties:

$$
\begin{aligned}
& \operatorname{tr}(a) \geq 0 \text { if } a \geq 0, \\
& \operatorname{tr}(a)=0 \text { and } a \geq 0 \text { implies } a=0, \\
& |\operatorname{tr}(a)| \leq \operatorname{tr}(|a|) \text { for all } a \in L_{1}(\mathcal{M}) .
\end{aligned}
$$

If $a \in L_{p}(\mathcal{M})$ has the polar decomposition $a=u|a|$, then $u \in \mathcal{M}$ and $|a| \in L_{p}(\mathcal{M})$. For $1 \leq p<\infty$, the norm on $L_{p}(\mathcal{M})$ is given by 


$$
\|a\|_{p}=\operatorname{tr}\left(|a|^{p}\right)^{\frac{1}{p}}, \quad a \in L_{p}(\mathcal{M}) .
$$

For $p=\infty$ and $a \in \mathcal{M}$ the norm $\|a\|_{\infty}$ is the usual operator norm. If $\frac{1}{p}+\frac{1}{q}=\frac{1}{r}, a \in$ $L_{p}(\mathcal{M})$ and $b \in L_{q}(\mathcal{M})$ then $a b \in L_{r}(\mathcal{M})$ and

$$
\|a b\|_{r} \leq\|a\|_{p}\|b\|_{q} .
$$

Especially, when $\frac{1}{p}+\frac{1}{q}=1$ we get for $a \in L_{p}(\mathcal{M})$ and $b \in L_{q}(\mathcal{M})$

$$
\begin{aligned}
& \operatorname{tr}(a b)=\operatorname{tr}(b a), \\
& |\operatorname{tr}(a b)| \leq\|a b\|_{1} \leq\|a\|_{p}\|b\|_{q}, \\
& \operatorname{tr}(a b) \geq 0 \text { for all } b \in L_{q}(\mathcal{M})_{+} \text {if and only if } a \geq 0 .
\end{aligned}
$$

Moreover, the space $L_{q}(\mathcal{M})$ is isometrically isomorphic to the dual space of $L_{p}(\mathcal{M})$. We denote this duality by

$$
\langle a, b\rangle=\operatorname{tr}(a b) \text { for } a \in L_{p}(\mathcal{M}) \text { and } b \in L_{q}(\mathcal{M}) .
$$

If $\mathcal{M}$ has a normal faithful semifinite trace $\tau$, we get the $L_{p}$-spaces $L_{p}(\mathcal{M}, \tau)=\left\{a \mid a\right.$ is $\tau$ - measurable, $\left.\tau\left(|a|^{p}\right)<\infty\right\}$. It is shown in [13], pp. $62-63$ that $L_{p}(\mathcal{M})$ is isomorphic to $\left\{a \otimes f_{p} \mid a \in L_{p}(\mathcal{M}, \tau), f_{p}: \mathbb{R} \rightarrow \mathbb{R}, s \mapsto \exp (s / p)\right\}$. Therefore we can switch to the easier to handle spaces $L_{p}(\mathcal{M}, \tau)$ for semifinite von Neumann algebras.

For $n \in \mathbb{N}$, we denote the complex $n \times n$ matrices by $M_{n}$ with the usual trace $T r$. If $a=\left[a_{i j}\right]$ is an $n \times n$ matrix of $\tau$-measurable operators and each $a_{i j}$ acts on the Hilbert space $\mathcal{K}=L_{2}(\mathbb{R}, \mathcal{H})$, then $a$ acts on the Hilbert space $\mathcal{K}^{n}$. The operator $a$ is densely defined, has a unique closure which we denote again by $a$, and is $\tau \otimes T r$ measurable. Especially, the elements of $L_{p}(\mathcal{M}) \otimes M_{n}$ are $\tau \otimes T r$-measurable operators. The elements of $L_{p}(\mathcal{M}) \otimes M_{n}$ have a natural ordering as being densely defined operators on the Hilbert space $\mathcal{K}^{n}$. The positive operators in $L_{p}(\mathcal{M})$ will be denoted by $L_{p}(\mathcal{M})_{+}$, and the positive operators in $L_{p}(\mathcal{M}) \otimes M_{n}$ will be denoted by $\left(L_{p}(\mathcal{M}) \otimes M_{n}\right)_{+}$. Following the description in [7], p. 70, $L_{p}(\mathcal{M}) \otimes M_{n}$ is linearly isomorphic to $L_{p}\left(\mathcal{M} \otimes M_{n}\right)$ and this isomorphism maps the positive cone $\left(L_{p}(\mathcal{M}) \otimes M_{n}\right)_{+}$to $L_{p}\left(\mathcal{M} \otimes M_{n}\right)_{+}$. Furthermore for $\frac{1}{p}+\frac{1}{p^{\prime}}=1$, this isomorphism defines the duality of $L_{p}(\mathcal{M}) \otimes M_{n}$ and $L_{p^{\prime}}(\mathcal{M}) \otimes M_{n}$ by

$$
\langle a, b\rangle=\sum_{i, j=1}^{n}\left\langle a_{i j}, b_{j i}\right\rangle, a=\left[a_{i j}\right] \in L_{p}(\mathcal{M}) \otimes M_{n}, b=\left[b_{i j}\right] \in L_{p^{\prime}}(\mathcal{M}) \otimes M_{n} .
$$

By (2.2),

$$
a \geq 0 \text { if and only if }\langle a, b\rangle \geq 0 \text { for all } b \in\left(L_{p^{\prime}}(\mathcal{M}) \otimes M_{n}\right)_{+} .
$$

Next, we describe some relationships between matrices and their elements. 
Lemma 2.1 Let $\mathcal{M}_{1}$ be a von Neumann algebra acting on a Hilbert space $\mathcal{H}$ with a normal faithful semifinite trace $\tau$, and let $a$ and $b$ be self-adjoint $\tau$-measurable operators. Then the following are equivalent:

(i) $-a \leq b \leq a$.

(ii) The matrix $\left[\begin{array}{ll}a & b \\ b & a\end{array}\right]$ is positive.

Proof We show first that (ii) implies (i): Let $\mathcal{D}(a)$ and $\mathcal{D}(b)$ denote the domains of $a$ and $b$. Let $\mathcal{D}=\mathcal{D}(a) \cap \mathcal{D}(b)$. Then $\mathcal{D}$ is a $\tau$-dense subspace of $\mathcal{H}$. For $\xi \in \mathcal{D}$, we get

$$
0 \leq \frac{1}{2}\left(\left[\begin{array}{ll}
a & b \\
b & a
\end{array}\right]\left[\begin{array}{l}
\xi \\
\xi
\end{array}\right] \mid\left[\begin{array}{l}
\xi \\
\xi
\end{array}\right]\right)=((a+b) \xi \mid \xi)
$$

Hence $-a \leq b$ on $\mathcal{D}$. By replacing the vector $\left[\begin{array}{l}\xi \\ \xi\end{array}\right]$ in $(2.4)$ with $\left[\begin{array}{c}\xi \\ -\xi\end{array}\right]$, we get $b \leq a$.

For the implication i) $\Rightarrow$ ii), we assume first that $a$ and $b$ are bounded. Then it follows from [3], Proposition 1.3.5 that $\left[\begin{array}{ll}a & b \\ b & a\end{array}\right]$ is positive. Since $\mathcal{D}(a) \cap \mathcal{D}(b)$ is $\tau$ dense, there is a sequence of projections $\left(p_{n}\right)_{n=1}^{\infty}$ in $\mathcal{M}_{1}$ such that $p_{n} \leq p_{n+1}$ for all $n \in \mathbb{N}, \tau\left(1-p_{n}\right) \rightarrow 0$ as $n \rightarrow \infty$, and $p_{n} \mathcal{H} \subseteq \mathcal{D}(a) \cap \mathcal{D}(b)$. Hence $p_{n} a p_{n}$ and $p_{n} b p_{n}$ are bounded operators and for all $n \in \mathbb{N}$

$$
-p_{n} a p_{n} \leq p_{n} b p_{n} \leq p a p_{n}
$$

Therefore, for $\xi, \eta \in p_{n} \mathcal{H}$, we have

$$
\begin{aligned}
\left(\left[\begin{array}{ll}
a & b \\
b & a
\end{array}\right]\left[\begin{array}{l}
\xi \\
\eta
\end{array}\right] \mid\left[\begin{array}{l}
\xi \\
\eta
\end{array}\right]\right) & =\left(\left[\begin{array}{ll}
a & b \\
b & a
\end{array}\right]\left[\begin{array}{l}
p_{n} \xi \\
p_{n} \eta
\end{array}\right] \mid\left[\begin{array}{l}
p_{n} \xi \\
p_{n} \eta
\end{array}\right]\right) \\
& =\left(\left[\begin{array}{ll}
p_{n} a p_{n} & p_{n} b p_{n} \\
p_{n} b p_{n} & p_{n} a p_{n}
\end{array}\right]\left[\begin{array}{l}
\xi \\
\eta
\end{array}\right] \mid\left[\begin{array}{l}
\xi \\
\eta
\end{array}\right]\right) \geq 0 .
\end{aligned}
$$

Since the union $\bigcup_{n \in \mathbb{N}} p_{n} \mathcal{H}$ is $\tau$-dense, $\left[\begin{array}{ll}a & b \\ b & a\end{array}\right]$ is positive.

For $n \in \mathbb{N}, 1_{n}$ denotes the unit matrix in $M_{n}$. For a $\tau$-measurable operator $a$ let $\operatorname{supp}(a)$ denote the smallest projection which fulfills $\operatorname{supp}(a) \cdot a=a \cdot \operatorname{supp}(a)=a$. If $a \in L_{p}(\mathcal{M})$ then $\operatorname{supp}(a) \in \mathcal{M}$ although $a$ is $\tau$-measurable with respect to a larger algebra (see [13], Proposition II.4).

Lemma 2.2 Let $\mathcal{M}_{1}$ be a von Neumann algebra with a normal faithful semifinite trace $\tau$. Let $a, b, c_{1}, c_{2}$ be $\tau$-measurable operators for which holds:

(i) The operators $a$ and $b$ are positive.

(ii) $\operatorname{supp}(a) \cdot c_{1} \cdot \operatorname{supp}(b)=c_{1}$ and $\operatorname{supp}(a) \cdot c_{2} \cdot \operatorname{supp}(b)=c_{2}$.

(iii) $a c_{1} b=a c_{2} b$. 
Then $c_{1}=c_{2}$.

Proof By putting $c=c_{1}-c_{2}$, we may assume that $c_{2}=0$. First let $a=b$. Then ii) can be formulated as $\operatorname{supp}(a) \cdot c \cdot \operatorname{supp}(a)=c$ and iii) as $a c a=0$. This means that the left support and the right support of $c$ are less than or equal to $\operatorname{supp}(a)$. Thus $c$ fulfills the conditions of [11], Lemma 2.2 (c), and therefore $c=0$. For the general case, we put $a^{\prime}=\left[\begin{array}{ll}a & 0 \\ 0 & b\end{array}\right]$ and $c^{\prime}=\left[\begin{array}{ll}0 & c \\ 0 & 0\end{array}\right]$. Then

$$
\begin{array}{r}
\operatorname{supp}\left(a^{\prime}\right)=\left[\begin{array}{cc}
\operatorname{supp}(a) & 0 \\
0 & \operatorname{supp}(b)
\end{array}\right], \\
a^{\prime} c^{\prime} a^{\prime}=\left[\begin{array}{ll}
a & 0 \\
0 & b
\end{array}\right]\left[\begin{array}{ll}
0 & c \\
0 & 0
\end{array}\right]\left[\begin{array}{ll}
a & 0 \\
0 & b
\end{array}\right]=\left[\begin{array}{cc}
0 & a c b \\
0 & 0
\end{array}\right]=0,
\end{array}
$$

and

$$
\operatorname{supp}\left(a^{\prime}\right) \cdot c^{\prime} \cdot \operatorname{supp}\left(a^{\prime}\right)=\left[\begin{array}{cc}
0 & \operatorname{supp}(a) \cdot c \cdot \operatorname{supp}(b) \\
0 & 0
\end{array}\right]=0 .
$$

Hence, by the first part of the proof, $c^{\prime}=0$ which implies $c=0$.

Theorem 2.3 Let $\mathcal{M}$ be a von Neumann algebra with a normal faithful semifinite weight $\varphi$ and acting on the Hilbert space $\mathcal{H}, 1 \leq p \leq \infty, n \in \mathbb{N}, f, g \in L_{p}(\mathcal{M})_{+}$, and $x \in L_{p}(\mathcal{M}) \otimes M_{n}$ such that

$$
\left[\begin{array}{cc}
f \otimes 1_{n} & x \\
x^{*} & g \otimes 1_{n}
\end{array}\right] \geq 0 .
$$

Then there is an operator $y \in \mathcal{M} \otimes M_{n}$ such that

$$
x=\left(f^{\frac{1}{2}} \otimes 1_{n}\right) y\left(g^{\frac{1}{2}} \otimes 1_{n}\right) .
$$

We have $\|y\|_{\infty} \leq 1$ and $y$ is unique subject to the condition $\left(\operatorname{supp}(f) \otimes 1_{n}\right) \cdot y$. $\left(\operatorname{supp}(g) \otimes 1_{n}\right)=y$ and to (2.5). If $f=g$ and $x$ is self-adjoint, then $y$ is self-adjoint. If $f=g$ and $x$ is positive, then $y$ is positive.

Proof Let $\mathcal{M}_{1}=\mathcal{M} \rtimes_{\sigma^{\varphi}} \mathbb{R}, \mathcal{K}=L_{2}(\mathbb{R}, \mathcal{H})$, and $\tau$ be the canonical trace on $\mathcal{M}_{1}$. Let $x=\left[x_{i j}\right]$ and

$$
\mathcal{D}=\mathcal{D}(f) \cap \mathcal{D}\left(f^{\frac{1}{2}}\right) \cap \mathcal{D}(g) \cap \mathcal{D}\left(g^{\frac{1}{2}}\right) \cap \bigcap_{i, j=1}^{n} \mathcal{D}\left(x_{i j}\right) .
$$

Then $\mathcal{D}$ is $\tau$-dense in $\mathcal{K}$ and consequently, $\mathcal{D}^{n}$ is $\tau \otimes \operatorname{Tr}$-dense in $\mathcal{K}^{n}$. For $\xi, \eta \in \mathcal{D}^{n}$, we get 


$$
\begin{aligned}
0 & \leq\left(\left[\begin{array}{cc}
f \otimes 1_{n} & x \\
x^{*} & g \otimes 1_{n}
\end{array}\right]\left[\begin{array}{l}
\xi \\
\eta
\end{array}\right] \mid\left[\begin{array}{l}
\xi \\
\eta
\end{array}\right]\right) \\
& =\left(\left(f \otimes 1_{n}\right) \xi \mid \xi\right)+(x \eta \mid \xi)+\left(x^{*} \xi \mid \eta\right)+\left(\left(g \otimes 1_{n}\right) \eta \mid \eta\right) .
\end{aligned}
$$

This implies

$$
-2 \operatorname{Re}((x \eta \mid \xi)) \leq\left(\left(f \otimes 1_{n}\right) \xi \mid \xi\right)+\left(\left(g \otimes 1_{n}\right) \eta \mid \eta\right) .
$$

We replace $\eta$ in (2.6) by $e^{\text {it }} \eta$, and choose a suitable value for $t \in \mathbb{R}$ to get

$$
2|(x \eta \mid \xi)| \leq\left(\left(f \otimes 1_{n}\right) \xi \mid \xi\right)+\left(\left(g \otimes 1_{n}\right) \eta \mid \eta\right) .
$$

Then we replace $\xi$ by $\lambda \xi, \eta$ by $\frac{1}{\lambda} \eta$ in (2.7), minimize over $\lambda \in \mathbb{R}_{+}$, and get

$$
|(x \eta \mid \xi)|^{2} \leq\left(\left(f \otimes 1_{n}\right) \xi \mid \xi\right)\left(\left(g \otimes 1_{n}\right) \eta \mid \eta\right) .
$$

So, we can define the sesquilinear form

$$
\begin{aligned}
B: & \left(g^{\frac{1}{2}} \otimes 1_{n}\right) \mathcal{D}^{n} \times\left(f^{\frac{1}{2}} \otimes 1_{n}\right) \mathcal{D}^{n} \rightarrow \mathbb{C} \\
& \left(\left(g^{\frac{1}{2}} \otimes 1_{n}\right) \eta,\left(f^{\frac{1}{2}} \otimes 1_{n}\right) \xi\right) \mapsto(x \eta \mid \xi) .
\end{aligned}
$$

If $\xi, \xi^{\prime}, \eta, \eta^{\prime} \in \mathcal{D}^{n}$ with $\left(f^{\frac{1}{2}} \otimes 1_{n}\right) \xi=\left(f^{\frac{1}{2}} \otimes 1_{n}\right) \xi^{\prime}$ and $\left(g^{\frac{1}{2}} \otimes 1_{n}\right) \eta=\left(g^{\frac{1}{2}} \otimes 1_{n}\right) \eta^{\prime}$, we get

$$
\begin{aligned}
\left|(x \eta \mid \xi)-\left(x \eta^{\prime} \mid \xi^{\prime}\right)\right| \leq & \left|\left(x \eta \mid \xi-\xi^{\prime}\right)\right|+\left|\left(x\left(\eta-\eta^{\prime}\right) \mid \xi^{\prime}\right)\right| \\
\leq & \left(\left(f \otimes 1_{n}\right)\left(\xi-\xi^{\prime}\right) \mid \xi-\xi^{\prime}\right)^{\frac{1}{2}}\left(\left(g \otimes 1_{n}\right) \eta \mid \eta\right)^{\frac{1}{2}} \\
& +\left(\left(f \otimes 1_{n}\right) \xi^{\prime} \mid \xi^{\prime}\right)^{\frac{1}{2}}\left(\left(g \otimes 1_{n}\right)\left(\eta-\eta^{\prime}\right) \mid \eta-\eta^{\prime}\right)^{\frac{1}{2}} \\
= & 0 .
\end{aligned}
$$

This shows that $B$ is well defined, and by (2.8), we get

$$
\begin{aligned}
& \left|B\left(\left(g^{\frac{1}{2}} \otimes 1_{n}\right) \eta,\left(f^{\frac{1}{2}} \otimes 1_{n}\right) \xi\right)\right|^{2}=|(x \eta \mid \xi)|^{2} \\
& \quad \leq\left(\left(g^{\frac{1}{2}} \otimes 1_{n}\right) \eta \mid\left(g^{\frac{1}{2}} \otimes 1_{n}\right) \eta\right)\left(\left(f^{\frac{1}{2}} \otimes 1_{n}\right) \xi \mid\left(f^{\frac{1}{2}} \otimes 1_{n}\right) \xi\right) .
\end{aligned}
$$

Thus, $B$ can be extended to a bounded sesquilinear form

$$
B:\left(\operatorname{supp}(g) \otimes 1_{n}\right) \mathcal{K}^{n} \times\left(\operatorname{supp}(f) \otimes 1_{n}\right) \mathcal{K}^{n} \rightarrow \mathbb{C}
$$

with norm $\|B\| \leq 1$. Next we extend $B$ to a bounded sesquilinear form on $\mathcal{K}^{n} \times \mathcal{K}^{n}$ by $B(\eta, \xi)=B\left(\left(\operatorname{supp}(g) \otimes 1_{n}\right) \eta,\left(\operatorname{supp}(f) \otimes 1_{n}\right) \xi\right)$ for $\xi, \eta \in \mathcal{K}^{n}$. Then $B$ has still norm $\|B\| \leq 1$. Therefore, there is an operator $y \in \mathcal{B}\left(\mathcal{K}^{n}\right)$ with $(y \eta \mid \xi)=B(\eta, \xi)$ and $\|y\|_{\infty}=\|B\| \leq 1$. By construction, we have

$$
\left(\operatorname{supp}(f) \otimes 1_{n}\right) \cdot y \cdot\left(\operatorname{supp}(g) \otimes 1_{n}\right)=y .
$$

We still have to show that $y \in \mathcal{M} \otimes M_{n}$. Let $u \in \mathcal{M}_{1}^{\prime}$ be a unitary element of the 
commutant of $\mathcal{M}_{1}, \xi, \eta \in \mathcal{K}^{n}$. Then there are sequences $\left(\xi_{i}\right)_{i=1}^{\infty}$ and $\left(\eta_{i}\right)_{i=1}^{\infty}$ with $\xi_{i}, \eta_{i} \in \mathcal{D}^{n}$ for all $i \in \mathbb{N}$,

$$
\left(\operatorname{supp}(f) \otimes 1_{n}\right) \xi=\lim _{i \rightarrow \infty}\left(f^{\frac{1}{2}} \otimes 1_{n}\right) \xi_{i}
$$

and

$$
\left(\operatorname{supp}(g) \otimes 1_{n}\right) \eta=\lim _{i \rightarrow \infty}\left(g^{\frac{1}{2}} \otimes 1_{n}\right) \eta_{i}
$$

Since $u$ commutes with $f, g, \operatorname{supp}(f)$, and $\operatorname{supp}(g)$, we get for all $i \in \mathbb{N}$

$$
\begin{aligned}
& \left(\left(u^{*} \otimes 1_{n}\right) y\left(u \otimes 1_{n}\right)\left(g^{\frac{1}{2}} \otimes 1_{n}\right) \eta_{i} \mid\left(f^{\frac{1}{2}} \otimes 1_{n}\right) \xi_{i}\right) \\
& =\left(\left(u^{*} \otimes 1_{n}\right)\left(f^{\frac{1}{2}} \otimes 1_{n}\right) y\left(g^{\frac{1}{2}} \otimes 1_{n}\right)\left(u \otimes 1_{n}\right) \eta_{i} \mid \xi_{i}\right) \\
& =\left(\left(u^{*} \otimes 1_{n}\right) x\left(u \otimes 1_{n}\right) \eta_{i} \mid \xi_{i}\right) \\
& =\left(x \eta_{i} \mid \xi_{i}\right) \\
& =\left(\left(f^{\frac{1}{2}} \otimes 1_{n}\right) y\left(g^{\frac{1}{2}} \otimes 1_{n}\right) \eta_{i} \mid \xi_{i}\right)
\end{aligned}
$$

and therefore

$$
\begin{aligned}
& \left(\left(u^{*} \otimes 1_{n}\right) y\left(u \otimes 1_{n}\right) \eta \mid \xi\right) \\
& =\left(\left(u^{*} \otimes 1_{n}\right)\left(\operatorname{supp}(f) \otimes 1_{n}\right) y\left(\operatorname{supp}(g) \otimes 1_{n}\right)\left(u \otimes 1_{n}\right) \eta \mid \xi\right) \\
& =\left(\left(u^{*} \otimes 1_{n}\right) y\left(u \otimes 1_{n}\right)\left(\operatorname{supp}(g) \otimes 1_{n}\right) \eta \mid\left(\operatorname{supp}(f) \otimes 1_{n}\right) \xi\right) \\
& =\lim _{i \rightarrow \infty}\left(\left(u^{*} \otimes 1_{n}\right) y\left(u \otimes 1_{n}\right)\left(g^{\frac{1}{2}} \otimes 1_{n}\right) \eta_{i} \mid\left(f^{\frac{1}{2}} \otimes 1_{n}\right) \xi_{i}\right) \\
& =\lim _{i \rightarrow \infty}\left(\left(f^{\frac{1}{2}} \otimes 1_{n}\right) y\left(g^{\frac{1}{2}} \otimes 1_{n}\right) \eta_{i} \mid \xi_{i}\right) \\
& =(y \eta \mid \xi) .
\end{aligned}
$$

Every operator in $\mathcal{M}_{1}^{\prime}$ can be written as a finite linear combination of unitaries and $\left(\mathcal{M}_{1} \otimes M_{n}\right)^{\prime}=\mathcal{M}_{1}^{\prime} \otimes \mathbb{C}$. Hence $y \in \mathcal{M}_{1} \otimes M_{n}$. We still have to show that $y \in \mathcal{M} \otimes M_{n}$. Let $y=\left[y_{i j}\right]$ with $y_{i j} \in \mathcal{M}_{1}$ for $i, j \in\{1, \ldots n\}$. Let $s \in \mathbb{R}$. Then we have

$$
\begin{aligned}
f^{\frac{1}{2}} y_{i j} g^{\frac{1}{2}} & =x_{i j}=e^{\frac{s}{p}} \theta_{s}\left(x_{i j}\right) \\
& =e^{\frac{s}{p}} \theta_{s}\left(f^{\frac{1}{2}}\right) \theta_{s}\left(y_{i j}\right) \theta_{s}\left(g^{\frac{1}{2}}\right)=f^{\frac{1}{2}} \theta_{s}\left(y_{i j}\right) g^{\frac{1}{2}} .
\end{aligned}
$$

Since

$$
y_{i j}=\operatorname{supp}(f) \cdot y_{i j} \cdot \operatorname{supp}(g)
$$

and 


$$
\theta_{s}\left(y_{i j}\right)=\operatorname{supp}(f) \cdot \theta_{s}\left(y_{i j}\right) \cdot \operatorname{supp}(g),
$$

we can apply Lemma 2.2 and get $y_{i j}=\theta_{s}\left(y_{i j}\right)$. Hence $y_{i j} \in \mathcal{M}$. For the uniqueness of the decomposition, suppose that there is another $\tilde{y} \in \mathcal{M} \otimes M_{n}$ such that

$$
x=\left(f^{\frac{1}{2}} \otimes 1_{n}\right) \tilde{y}\left(g^{\frac{1}{2}} \otimes 1_{n}\right)
$$

and

$$
\left(\operatorname{supp}(f) \otimes 1_{n}\right) \cdot \tilde{y} \cdot\left(\operatorname{supp}(g) \otimes 1_{n}\right)=\tilde{y} .
$$

Then we can apply Lemma 2.2 and get $y=\tilde{y}$. Now assume that $x$ is self-adjoint and $f=g$. Since $x=x^{*}$, we get

$$
\begin{aligned}
\left(f^{\frac{1}{2}} \otimes 1_{n}\right) y\left(f^{\frac{1}{2}} \otimes 1_{n}\right) & =x=\frac{1}{2}\left(x+x^{*}\right) \\
& =\left(f^{\frac{1}{2}} \otimes 1_{n}\right) \frac{1}{2}\left(y+y^{*}\right)\left(f^{\frac{1}{2}} \otimes 1_{n}\right) .
\end{aligned}
$$

Again, we apply Lemma 2.2, use the uniqueness of $y$, and get $y=y^{*}$. Finally, assume that $x$ is positive. For $\xi \in \mathcal{K}^{n}$ there is a sequence $\left(\xi_{i}\right)_{i=1}^{\infty}$ with $\xi_{i} \in \mathcal{D}^{n}$ for all $i \in \mathbb{N}$ and

$$
\left(\operatorname{supp}(f) \otimes 1_{n}\right) \xi=\lim _{i \rightarrow \infty}\left(f^{\frac{1}{2}} \otimes 1_{n}\right) \xi_{i}
$$

Then

$$
\begin{aligned}
(y \xi \mid \xi) & =\left(y\left(\operatorname{supp}(f) \otimes 1_{n}\right) \xi \mid\left(\operatorname{supp}(f) \otimes 1_{n}\right) \xi\right) \\
& =\lim _{i \rightarrow \infty}\left(y\left(f^{\frac{1}{2}} \otimes 1_{n}\right) \xi_{i}\left|\left(f^{\frac{1}{2}} \otimes 1_{n}\right) \xi_{i}\right| \xi_{i}\right) \\
& =\lim _{i \rightarrow \infty}\left(x \xi_{i} \mid \xi_{i}\right) \geq 0 .
\end{aligned}
$$

Since $\xi$ was arbitrary, $y$ is positive.

Lemma 2.4 Let $\mathcal{M}$ be a von Neumann algebra, $1 \leq p \leq \infty, n \in \mathbb{N}$, and $x \in L_{p}(\mathcal{M}) \otimes M_{n}$. Then there exists $f \in L_{p}(\mathcal{M})_{+}$such that

$$
\left[\begin{array}{cc}
f \otimes 1_{n} & x \\
x^{*} & f \otimes 1_{n}
\end{array}\right] \geq 0 .
$$

Proof Since every $x \in L_{p}(\mathcal{M}) \otimes M_{n}$ is a finite linear combination of elements of the form $y \otimes \alpha$, where $y \in L_{p}(\mathcal{M})_{+}$and $\alpha \in M_{n}$, it suffices to prove the statement for $x=y \otimes \alpha$. Let $\|\alpha\|$ denote the usual maximum norm of an $n \times n$ matrix acting on $\mathbb{C}^{n}$. Then we get 


$$
\begin{aligned}
& {\left[\begin{array}{cc}
\|\alpha\| y \otimes 1_{n} & y \otimes \alpha \\
y \otimes \alpha^{*} & \|\alpha\| y \otimes 1_{n}
\end{array}\right]} \\
& =\left[\begin{array}{cc}
y^{\frac{1}{2}} \otimes 1_{n} & 0 \\
0 & y^{\frac{1}{2}} \otimes 1_{n}
\end{array}\right]\left[\begin{array}{cc}
\|\alpha\| 1_{n} & \alpha \\
\alpha^{*} & \|\alpha\| 1_{n}
\end{array}\right]\left[\begin{array}{cc}
y^{\frac{1}{2}} \otimes 1_{n} & 0 \\
0 & y^{\frac{1}{2}} \otimes 1_{n}
\end{array}\right] \geq 0 .
\end{aligned}
$$

Lemma 2.5 Let $\mathcal{M}$ be a von Neumann algebra, $1 \leq p \leq \infty, n \in \mathbb{N}$, and $x \in\left(L_{p}(\mathcal{M}) \otimes M_{n}\right)_{+}$. Then $x$ is a finite sum of matrices of the form $\left[x_{i}^{*} x_{j}\right]$ where $x_{i} \in L_{2 p}(\mathcal{M})$ for $i \in\{1, \ldots, n\}$.

Proof By Lemmas 2.4 and 2.3, there exists $f \in L_{p}(\mathcal{M})_{+}$and $y \in\left(\mathcal{M} \otimes M_{n}\right)_{+}$such that $x=\left(f^{\frac{1}{2}} \otimes 1_{n}\right) y\left(f^{\frac{1}{2}} \otimes 1_{n}\right)$.. By applying [12], page 193, Lemma 3.1, to $y$ we get the desired result.

\section{Matrix norms on non-commutative $L_{p}$-spaces}

In this section $\mathcal{M}$ always denotes a von Neumann algebra without any further restrictions. For each $n \in \mathbb{N}$, we will define a norm on $L_{p}(\mathcal{M}) \otimes M_{n}$ and derive some properties of this norm.

Definition 3.1 Let $1 \leq p \leq \infty, n \in \mathbb{N}$, and $x \in L_{p}(\mathcal{M}) \otimes M_{n}$. Then we define

$$
\|x\|_{p, n}=\inf \left\{\frac{1}{2}\left(\|f\|_{p}+\|g\|_{p}\right) \mid f, g \in L_{p}(\mathcal{M})_{+},\left[\begin{array}{cc}
f \otimes 1_{n} & x \\
x^{*} & g \otimes 1_{n}
\end{array}\right] \geq 0\right\} .
$$

Remark 3.2 By Lemma 2.4, the set on the right side of (3.1) is not empty, and therefore, the infimum is well defined.

If $p=\infty$, the norm $\|x\|_{\infty, n}$ is identical to the usual operator norm of $x$ considered as a bounded operator on a Hilbert space.

The combination of this norm definition with Theorem 2.3 shows that this norm is quite similar to the norm used in [9], Eq. (1.5).

If $\quad x \in L_{p}(\mathcal{M}) \otimes M_{n}, x=\sum_{j=1}^{k} x_{j} \otimes \beta_{j} \quad$ and $\quad \alpha \in M_{n}$, then we have $\alpha x=\sum_{j=1}^{k} x_{j} \otimes \alpha \beta_{j}$ and $x \alpha=\sum_{j=1}^{k} x_{j} \otimes \beta_{j} \alpha$. For $n \in \mathbb{N}, i, j \in\{1, \ldots, n\}$ let

$\varepsilon_{i j}$ be the $n \times n$ matrix with 1 at position $(i, j)$ and 0 else.

Theorem 3.3 Let $1 \leq p \leq \infty$ and $n \in \mathbb{N}$. Then the following holds:

(i) If $x, y \in L_{p}(\mathcal{M}) \otimes M_{n}$, then $\|x+y\|_{p, n} \leq\|x\|_{p, n}+\|y\|_{p, n}$.

(ii) If $x \in L_{p}(\mathcal{M}) \otimes M_{n}$, then $\left\|x^{*}\right\|_{p, n}=\|x\|_{p, n}$.

(iii) If $x \in L_{p}(\mathcal{M}) \otimes M_{n}$ and $\alpha \in M_{n}$, then 


$$
\|\alpha x\|_{p, n} \leq\|\alpha\|\|x\|_{p . n} \text { and }\|x \alpha\|_{p, n} \leq\|\alpha\|\|x\|_{p, n} .
$$

(iv) If $x \in L_{p}(\mathcal{M}) \otimes M_{n}$ and $\lambda \in \mathbb{C}$, then $\|\lambda x\|_{p, n}=|\lambda|\|x\|_{p, n}$.

(v) If $x=\left[x_{i j}\right] \in L_{p}(\mathcal{M}) \otimes M_{n}$, then

$$
\max \left\{\left\|x_{i j}\right\|_{p} \mid 1 \leq i, j \leq n\right\} \leq\|x\|_{p, n} \leq \sum_{i, j=1}^{n}\left\|x_{i j}\right\|_{p} .
$$

(vi) If $x \in L_{p}(\mathcal{M}) \otimes M_{n}$ with $\|x\|_{p, n}=0$, then $x=0$.

Proof Let $x, y \in L_{p}(\mathcal{M}) \otimes M_{n}$, and $\varepsilon>0$. Then there exist $f_{1}, f_{2}, g_{1}, g_{2} \in L_{p}(\mathcal{M})_{+}$ such that

$$
\left[\begin{array}{cc}
f_{1} \otimes 1_{n} & x \\
x^{*} & g_{1} \otimes 1_{n}
\end{array}\right] \geq 0, \quad \frac{1}{2}\left(\left\|f_{1}\right\|_{p}+\left\|g_{1}\right\|_{p}\right) \leq\|x\|_{p, n}+\varepsilon,
$$

and

$$
\left[\begin{array}{cc}
f_{2} \otimes 1_{n} & y \\
y^{*} & g_{2} \otimes 1_{n}
\end{array}\right] \geq 0, \quad \frac{1}{2}\left(\left\|f_{2}\right\|_{p}+\left\|g_{2}\right\|_{p}\right) \leq\|y\|_{p, n}+\varepsilon .
$$

Then we get

$$
\left[\begin{array}{cc}
\left(f_{1}+f_{2}\right) \otimes 1_{n} & x+y \\
(x+y)^{*} & \left(g_{1}+g_{2}\right) \otimes 1_{n}
\end{array}\right] \geq 0
$$

and

$$
\|x+y\|_{p, n} \leq \frac{1}{2}\left(\left\|f_{1}+f_{2}\right\|_{p}+\left\|g_{1}+g_{2}\right\|_{p}\right) \leq\|x\|_{p, n}+\|y\|_{p, n}+2 \varepsilon .
$$

Since $\varepsilon$ is arbitrary, (i) is proved.

To prove (ii), let $x \in L_{p}(\mathcal{M}) \otimes M_{n}$ and $\varepsilon>0$. Then there exist $f, g \in L_{p}(\mathcal{M})_{+}$ such that

$$
\left[\begin{array}{cc}
f \otimes 1_{n} & x \\
x^{*} & g \otimes 1_{n}
\end{array}\right] \geq 0, \quad \frac{1}{2}\left(\|f\|_{p}+\|g\|_{p}\right) \leq\|x\|_{p, n}+\varepsilon .
$$

Then we get

$$
0 \leq\left[\begin{array}{ll}
0 & 1 \\
1 & 0
\end{array}\right]\left[\begin{array}{cc}
f \otimes 1_{n} & x \\
x^{*} & g \otimes 1_{n}
\end{array}\right]\left[\begin{array}{ll}
0 & 1 \\
1 & 0
\end{array}\right]=\left[\begin{array}{cc}
g \otimes 1_{n} & x^{*} \\
x & f \otimes 1_{n}
\end{array}\right] .
$$

Hence, we conclude $\left\|x^{*}\right\|_{p, n} \leq \frac{1}{2}\left(\|f\|_{p}+\|g\|_{p}\right) \leq\|x\|_{p, n}+\varepsilon$. Since $\varepsilon$ is arbitrary, we get $\left\|x^{*}\right\|_{p, n} \leq\|x\|_{p, n}$. Since $\|x\|_{p, n}=\left\|x^{* *}\right\|_{p, n} \leq\left\|x^{*}\right\|_{p, n}$, ii) is proved.

Next, we prove iii). Let $\alpha \in M_{n}$. If $\alpha=0$ then $\alpha x=0$ and the inequality is true. So let $\alpha \neq 0$. For $\varepsilon>0$, there exist $f, g \in L_{p}(\mathcal{M})_{+}$such that 


$$
\left[\begin{array}{cc}
f \otimes 1_{n} & x \\
x^{*} & g \otimes 1_{n}
\end{array}\right] \geq 0 \text { and } \frac{1}{2}\left(\|f\|_{p}+\|g\|_{p}\right) \leq\|x\|_{p, n}+\varepsilon .
$$

Then we have for $\lambda>0$

$$
\begin{aligned}
0 & \leq\left[\begin{array}{cc}
\frac{1}{\lambda} \alpha & 0 \\
0 & \lambda 1_{n}
\end{array}\right]\left[\begin{array}{cc}
f \otimes 1_{n} & x \\
x^{*} & g \otimes 1_{n}
\end{array}\right]\left[\begin{array}{cc}
\frac{1}{\lambda} \alpha^{*} & 0 \\
0 & \lambda 1_{n}
\end{array}\right] \\
& =\left[\begin{array}{cc}
\frac{1}{\lambda^{2}} \alpha \alpha^{*}\left(f \otimes 1_{n}\right) & \alpha x \\
(\alpha x)^{*} & \lambda^{2} g \otimes 1_{n}
\end{array}\right] \\
& \leq\left[\begin{array}{cc}
\frac{1}{\lambda^{2}}\|\alpha\|^{2} f \otimes 1_{n} & \alpha x \\
(\alpha x)^{*} & \lambda^{2} g \otimes 1_{n}
\end{array}\right] .
\end{aligned}
$$

We put $\lambda^{2}=\|\alpha\|$ and get

$$
\|\alpha x\|_{p, n} \leq \frac{1}{2}\left(\|\alpha\|\|f\|_{p}+\|\alpha\|\|g\|_{p}\right) \leq\|\alpha\|\left(\|x\|_{p, n}+\varepsilon\right) .
$$

Since $\varepsilon$ was arbitrary, we get the desired result. A similar argument proves the second inequality in iii).

To prove (iv), let $x \in L_{p}(\mathcal{M}) \otimes M_{n}$ and $\lambda \in \mathbb{C}$. If $\lambda=0$, we have

$$
\|\lambda x\|_{p, n}=0=|\lambda|\|x\|_{p, n} .
$$

For $\lambda \neq 0$, we put $\alpha=\lambda 1_{n}$, apply iii), and get

$$
\begin{array}{r}
\|\lambda x\|_{p, n}=\left\|\lambda 1_{n} x\right\|_{p, n} \leq|\lambda|\|x\|_{p, n} \text { and } \\
|\lambda|\|x\|_{p, n}=|\lambda|\left\|\frac{1}{\lambda} \lambda x\right\|_{p, n} \leq|\lambda| \frac{1}{|\lambda|}\|x\|_{p, n} \leq\|\lambda x\|_{p, n} .
\end{array}
$$

For v), let $x=\left[x_{i j}\right] \in L_{p}(\mathcal{M}) \otimes M_{n}$. For $\varepsilon>0$, there exist $f, g \in L_{p}(\mathcal{M})_{+}$such that

$$
\left[\begin{array}{cc}
f \otimes 1_{n} & x \\
x^{*} & g \otimes 1_{n}
\end{array}\right] \geq 0, \quad \frac{1}{2}\left(\|f\|_{p}+\|g\|_{p}\right) \leq\|x\|_{p, n}+\varepsilon .
$$

By Theorem 2.3, there exists $y \in \mathcal{M} \otimes M_{n}$, such that $\|y\|_{\infty} \leq 1$ and $x=\left(f^{\frac{1}{2}} \otimes 1_{n}\right) y\left(g^{\frac{1}{2}} \otimes 1_{n}\right)$. Then $\quad x_{i j}=f^{\frac{1}{2}} y_{i j} g^{\frac{1}{2}} \quad$ and $\quad\left\|y_{i j}\right\|_{\infty} \leq\|y\|_{\infty} \leq 1$ for all $i, j \in\{1, \ldots, n\}$. Hence

$$
\begin{aligned}
\left\|x_{i j}\right\|_{p} & =\left\|f^{\frac{1}{2}} y_{i j} g^{\frac{1}{2}}\right\|_{p} \leq\left\|f^{\frac{1}{2}}\right\|_{2 p}\left\|g^{\frac{1}{2}}\right\|_{2 p}=\sqrt{\|f\|_{p}\|g\|_{p}} \\
& \leq \frac{1}{2}\left(\|f\|_{p}+\|g\|_{p}\right) \leq\|x\|_{p, n}+\varepsilon, \quad i, j \in\{1, \ldots, n\} .
\end{aligned}
$$

For the second inequality of $v)$, let $y \in L_{p}(\mathcal{M})$ with polar decomposition $y=v|y|$. Then $\left|y^{*}\right| v=v|y|, v^{*}\left|y^{*}\right| v=|y|$, and 


$$
0 \leq\left[\begin{array}{cc}
1 & 0 \\
v^{*} & 0
\end{array}\right]\left[\begin{array}{cc}
\left|y^{*}\right| & 0 \\
0 & \left|y^{*}\right|
\end{array}\right]\left[\begin{array}{ll}
1 & v \\
0 & 0
\end{array}\right]=\left[\begin{array}{cc}
\left|y^{*}\right| & y \\
y^{*} & |y|
\end{array}\right]
$$

Now it follows that

$$
\left[\begin{array}{cc}
\left|y^{*}\right| \otimes 1_{n} & y \otimes 1_{n} \\
y^{*} \otimes 1_{n} & |y| \otimes 1_{n}
\end{array}\right] \geq 0
$$

and therefore $\left\|y \otimes 1_{n}\right\|_{p, n} \leq\|y\|_{p}$. For $i \in\{1, \ldots, n\}$, let $\varepsilon_{i j}$ be as in (3.2). Then we get for $x=\left[x_{i j}\right] \in L_{p}(\mathcal{M}) \otimes M_{n}$

$$
\|x\|_{p, n}=\left\|\sum_{i, j=1}^{n} x_{i j} \otimes \varepsilon_{i j}\right\|_{p, n} \leq \sum_{i, j=1}^{n}\left\|x_{i j} \otimes 1_{n}\right\|_{p, n}\left\|\varepsilon_{i j}\right\| \leq \sum_{i, j=1}^{n}\left\|x_{i j}\right\|_{p} .
$$

To prove (vi), let $\|x\|_{p, n}=0$. From (v), it follows that $x_{i j}=0$ for all $i, j \in\{1, \ldots, n\}$. Hence $x=0$.

The next theorem shows that the infimum in Definition 3.1 is actually a minimum.

Theorem 3.4 Let $1 \leq p \leq \infty, n \in \mathbb{N}$, and $x \in L_{p}(\mathcal{M}) \otimes M_{n}$. Then there exist $f, g \in L_{p}(\mathcal{M})_{+}$such that

$$
\left[\begin{array}{cc}
f \otimes 1_{n} & x \\
x^{*} & g \otimes 1_{n}
\end{array}\right] \geq 0 \text { and }\|f\|_{p}=\|g\|_{p}=\|x\|_{p, n} .
$$

Proof If $x=0$, we can take $f=g=0$. So suppose that $x \neq 0$. Let $1 \leq q \leq \infty$ and $\frac{1}{p}+\frac{1}{q}=1$. Let $L_{q}(\mathcal{M})^{*}$ be the dual space of $L_{q}(\mathcal{M})$. Note that $L_{q}(\mathcal{M})^{*}$ is $L_{p}(\mathcal{M})$ when $q<\infty$ and $\mathcal{M}^{*}$ when $q=\infty$. For $\varepsilon>0$ we define

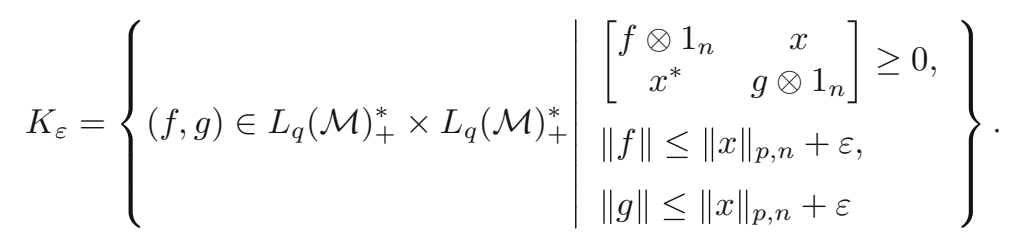

The symbol $\|\cdot\|$ denotes the norm of $L_{q}(\mathcal{M})^{*}$. The sets $K_{\varepsilon}, \varepsilon>0$, have the following properties:

Each $K_{\varepsilon} \neq \emptyset$ : By definition of $\|x\|_{p, n}$, there exist $0 \neq f, g \in L_{p}(\mathcal{M})_{+}$with

$$
\left[\begin{array}{cc}
f \otimes 1_{n} & x \\
x^{*} & g \otimes 1_{n}
\end{array}\right] \geq 0 \text { and } \frac{1}{2}\left(\|f\|_{p}+\|g\|_{p}\right)<\|x\|_{p, n}+\varepsilon .
$$

For $\lambda>0$, we get 


$$
0 \leq\left[\begin{array}{cc}
\lambda & 0 \\
0 & \frac{1}{\lambda}
\end{array}\right]\left[\begin{array}{cc}
f \otimes 1_{n} & x \\
x^{*} & g \otimes 1_{n}
\end{array}\right]\left[\begin{array}{cc}
\lambda & 0 \\
0 & \frac{1}{\lambda}
\end{array}\right]=\left[\begin{array}{cc}
\lambda^{2} f \otimes 1_{n} & x \\
x^{*} & \frac{1}{\lambda^{2}} g \otimes 1_{n}
\end{array}\right]
$$

We put $\lambda^{2}=\sqrt{\frac{\|g\|_{p}}{\|f\|_{p}}}, f^{\prime}=\lambda^{2} f$, and $g^{\prime}=\frac{1}{\lambda^{2}} g$. Then

$$
\left\|f^{\prime}\right\|_{p}=\left\|g^{\prime}\right\|_{p}=\sqrt{\|f\|_{p}\|g\|_{p}} \leq \frac{1}{2}\left(\|f\|_{p}+\|g\|_{p}\right)<\|x\|_{p, n}+\varepsilon
$$

and

$$
\left[\begin{array}{cc}
f^{\prime} \otimes 1_{n} & x \\
x^{*} & g^{\prime} \otimes 1_{n}
\end{array}\right] \geq 0 .
$$

This shows that $\left(f^{\prime}, g^{\prime}\right) \in K_{\varepsilon}$. Next we show that $K_{\varepsilon}$ is weak ${ }^{*}$-closed for every $\varepsilon>0$. We fix $\varepsilon>0$ and $(f, g)$ as an element of the weak* closure of $K_{\varepsilon}$. For $a=\left[\begin{array}{ll}a_{11} & a_{12} \\ a_{21} & a_{22}\end{array}\right] \in\left(L_{q}(\mathcal{M}) \otimes M_{2 n}\right)_{+}$there exist sequences $\left(f_{m}\right)_{m=1}^{\infty}$ and $\left(g_{m}\right)_{m=1}^{\infty}$ in $K_{\varepsilon}$ such that

$$
\left\langle f \otimes 1_{n}, a_{11}\right\rangle=\lim _{m \rightarrow \infty}\left\langle f_{m} \otimes 1_{n}, a_{11}\right\rangle
$$

and

$$
\left\langle g \otimes 1_{n}, a_{22}\right\rangle=\lim _{m \rightarrow \infty}\left\langle g_{m} \otimes 1_{n}, a_{22}\right\rangle,
$$

and $\left(f_{m}, g_{m}\right) \in K_{\varepsilon}$ for all $m \in \mathbb{N}$. Hence, we get

$$
\left\langle\left[\begin{array}{cc}
f \otimes 1_{n} & x \\
x^{*} & g \otimes 1_{n}
\end{array}\right] \mid a\right\rangle=\lim _{m \rightarrow \infty}\left\langle\left[\begin{array}{cc}
f_{m} \otimes 1_{n} & x \\
x^{*} & g_{m} \otimes 1_{n}
\end{array}\right] \mid a\right\rangle \geq 0 .
$$

Since this holds for every $a \in\left(L_{q}(\mathcal{M}) \otimes M_{2 n}\right)_{+}$, we can apply (2.3), and conclude that

$$
\left[\begin{array}{cc}
f \otimes 1_{n} & x \\
x^{*} & g \otimes 1_{n}
\end{array}\right] \geq 0 .
$$

If $q=\infty$, we have to do this in $\mathcal{M}^{*}$ which is isomorphic to $L_{1}\left(\mathcal{M}^{* *}\right)$. Especially, $f$ and $g$ are positive. Taking $a \in L_{q}(\mathcal{M})$ with $\|a\|_{q} \leq 1$, we can find a sequence $\left(f_{m}\right)_{m=1}^{\infty}$ in $K_{\varepsilon}$ such that

$$
\langle f, a\rangle=\lim _{m \rightarrow \infty}\left\langle f_{m}, a\right\rangle
$$

Hence $|\langle f, a\rangle| \leq\|x\|_{p, n}+\varepsilon$ and

$$
\|f\|=\sup \left\{|\langle f, a\rangle|\|a\|_{q} \leq 1\right\} \leq\|x\|_{p, n}+\varepsilon .
$$

Similarly, $\|g\| \leq\|x\|_{p, n}+\varepsilon$ and therefore $(f, g) \in K_{\varepsilon}$. By the Banach-Alaoglu 
theorem, the unit ball of $L_{q}(\mathcal{M})$ is compact in the weak*-topology. Hence all sets $K_{\varepsilon}$ are compact in the weak*-topology.

The sets $K_{\varepsilon}, \varepsilon>0$, have the finite intersection property: Given $k \in \mathbb{N}$, $\varepsilon_{1}, \ldots, \varepsilon_{k}>0$, we put $\varepsilon=\min \left\{\varepsilon_{1}, \ldots, \varepsilon_{k}\right\}$ and get

$$
K_{\varepsilon} \subseteq \bigcap_{i=1}^{k} K_{\varepsilon_{i}}
$$

Combining the finite intersection property and the weak-* compactness, we get

$$
\bigcap_{\varepsilon>0} K_{\varepsilon} \neq \emptyset
$$

Let $(f, g)$ be in the set defined in Eq. (3.4). Then we have

$$
\left[\begin{array}{cc}
f \otimes 1_{n} & x \\
x^{*} & g \otimes 1_{n}
\end{array}\right] \geq 0
$$

If $q<\infty$, then $f, g \in L_{p}(\mathcal{M})$. If $q=\infty$, there is a central projection in $\mathcal{M}^{* *}$ which works as projection from $\mathcal{M}^{*}$ to $L_{1}(\mathcal{M})$. Hence we may assume that $f, g \in L_{1}(\mathcal{M})$. By construction, we have $\|f\|_{p},\|g\|_{p} \leq\|x\|_{p, n}$. From Eq. (3.5), we get $2\|x\|_{p, n} \leq\|f\|_{p}+\|g\|_{p}$. Combining both gives

$$
\|f\|_{p}=\|x\|_{p, n}=\|g\|_{p} .
$$

Theorem 3.5 Let $1 \leq p \leq \infty, n \in \mathbb{N}$, and $x=x^{*} \in L_{p}(\mathcal{M}) \otimes M_{n}$.

(i) We have $\|x\|_{p, n}=\inf \left\{\|f\|_{p} \mid f \in L_{p}(\mathcal{M})_{+}, f \otimes 1_{n} \pm x \geq 0\right\}$.

(ii) There exists $f \in L_{p}(\mathcal{M})_{+}$such that

$$
f \otimes 1_{n} \pm x \geq 0 \text { and }\|f\|_{p}=\|x\|_{p, n} .
$$

Proof Let $A=\inf \left\{\|f\|_{p} \mid f \in L_{p}(\mathcal{M})_{+}, f \otimes 1_{n} \pm x \geq 0\right\}$. By Theorem 3.4, there exist $f, g \in L_{p}(\mathcal{M})_{+}$such that

$$
\|f\|_{p}=\|g\|_{p}=\|x\|_{p, n} \text { and }\left[\begin{array}{cc}
f \otimes 1_{n} & x \\
x & g \otimes 1_{n}
\end{array}\right] \geq 0 .
$$

It follows then

$$
0 \leq\left[\begin{array}{ll}
0 & 1 \\
1 & 0
\end{array}\right]\left[\begin{array}{cc}
f \otimes 1_{n} & x \\
x & g \otimes 1_{n}
\end{array}\right]\left[\begin{array}{ll}
0 & 1 \\
1 & 0
\end{array}\right]=\left[\begin{array}{cc}
g \otimes 1_{n} & x \\
x & f \otimes 1_{n}
\end{array}\right]
$$

Hence we conclude 


$$
\left[\begin{array}{cc}
\frac{1}{2}(f+g) \otimes 1_{n} & x \\
x & \frac{1}{2}(f+g) \otimes 1_{n}
\end{array}\right] \geq 0 .
$$

Then Lemma 2.1 implies that $\frac{1}{2}(f+g) \otimes 1_{n} \pm x \geq 0$. This shows that

$$
A \leq\left\|\frac{1}{2}(f+g)\right\|_{p} \leq\|x\|_{p, n} .
$$

For the converse direction, let $\varepsilon>0$. Then there exist $f \in L_{p}(\mathcal{M})_{+}$such that $f \otimes 1_{n} \pm x \geq 0$ and $\|f\|_{p} \leq A+\varepsilon$. It follows from Lemma 2.1 that

$$
\left[\begin{array}{cc}
f \otimes 1_{n} & x \\
x & f \otimes 1_{n}
\end{array}\right] \geq 0 .
$$

Hence, we get $\|x\|_{p, n} \leq\|f\|_{p} \leq A+\varepsilon$. Since $\varepsilon$ is arbitrary, we get $\|x\|_{p, n} \leq A$. This proves (i). To prove (ii), we take $f$ and $g$ from (3.6). Then we have

$$
\frac{1}{2}(f+g) \pm x \geq 0
$$

and

$$
\|x\|_{p, n} \leq \frac{1}{2}\|f+g\|_{p} \leq \frac{1}{2}\left(\|f\|_{p}+\|g\|_{p}\right)=\|x\|_{p, n} .
$$

This shows that $\left\|\frac{1}{2}(f+g)\right\|_{p}=\|x\|_{p, n}$.

Theorem 3.6 Let $1 \leq p \leq \infty$ and $x \in L_{p}(\mathcal{M})$. Then

$$
\|x\|_{p, 1}=\|x\|_{p} .
$$

Proof Let $x=v|x|$ be the polar decomposition of $x$. By Eq. (3.3), we get $\left[\begin{array}{cc}\left|x^{*}\right| & x \\ x^{*} & |x|\end{array}\right] \geq 0$. Hence,

$$
\|x\|_{p, 1} \leq \frac{1}{2}\left(\|x\|_{p}+\left\|x^{*}\right\|_{p}\right)=\|x\|_{p}
$$

To prove the converse inequality, we apply Theorems 3.4 and 2.3 and get $f, g \in L_{p}(\mathcal{M})_{+},\|f\|_{p}=\|g\|_{p}=\|x\|_{p, 1}, y \in \mathcal{M},\|y\|_{\infty} \leq 1$ and $x=f^{\frac{1}{2}} y g^{\frac{1}{2}}$. Hence

$$
\|x\|_{p}=\left\|f^{\frac{1}{2}} y g^{\frac{1}{2}}\right\|_{p} \leq\|f\|_{p}^{\frac{1}{2}}\|y\|_{\infty}\|g\|_{p}^{\frac{1}{2}}=\|x\|_{p, 1} .
$$




\section{Completely order bounded maps}

In this section, we define completely order bounded maps from $L_{p}$ to $L_{q}$ and show the decomposition of such maps for $p=\infty, q$ arbitrary and for $p$ arbitrary, $q=1$. For $2 p<q<\infty$ we give an example of a completely order bounded map which is not decomposable.

Throughout this Sects. $\mathcal{M}$ and $\mathcal{N}$ are von Neumann algebras with no further restrictions unless stated explicitly. If $1 \leq p, q \leq \infty, n \in \mathbb{N}$, and $T: L_{p}(\mathcal{M}) \rightarrow L_{q}(\mathcal{N})$ is a linear map, then

$$
T_{n}: L_{p}(\mathcal{M}) \otimes M_{n} \rightarrow L_{q}(\mathcal{N}) \otimes M_{n},\left[x_{i j}\right] \longmapsto\left[T\left(x_{i j}\right)\right] .
$$

We need the notion of decomposable maps which were introduced for $C^{*}$-algebras in [5] and extended to non-commutative $L_{p}$-spaces in [6,9]. The above map $T$ is called decomposable if there exist completely positive maps $S_{1}, S_{2}: L_{p}(\mathcal{M}) \rightarrow L_{q}(\mathcal{N})$ such that the induced map

$$
\Phi: L_{p}(\mathcal{M}) \otimes M_{2} \rightarrow L_{q}(\mathcal{N}) \otimes M_{2},\left[\begin{array}{ll}
x_{11} & x_{12} \\
x_{21} & x_{22}
\end{array}\right] \mapsto\left[\begin{array}{ll}
S_{1}\left(x_{11}\right) & T\left(x_{12}\right) \\
T\left(x_{21}^{*}\right)^{*} & S_{2}\left(x_{22}\right)
\end{array}\right]
$$

is completely positive. The decomposable norm $\|T\|_{\text {dec }}$ is defined by

$$
\|T\|_{d e c}=\inf \left\{\max \left\{\left\|S_{1}\right\|,\left\|S_{2}\right\|\right\}\right\}
$$

where the infimum is taken over all completely positive maps $S_{1}$ and $S_{2}$ in (4.1).

Definition 4.1 Let $1 \leq p, q \leq \infty$. A linear map $T: L_{p}(\mathcal{M}) \rightarrow L_{q}(\mathcal{N})$ is called completely order bounded, if

$$
\|T\|_{c o b}=\sup \left\{\left\|T_{n}(x)\right\|_{q, n} \mid x \in L_{p}(\mathcal{M}) \otimes M_{n},\|x\|_{p, n} \leq 1, n \in \mathbb{N}\right\}<\infty .
$$

The name completely order bounded will be justified by Theorem 4.5 where we show that a completely order bounded map maps order intervals to order intervals uniformly over all matrix levels.

Proposition 4.2 Let $1 \leq p, q \leq \infty$ and $T: L_{p}(\mathcal{M}) \rightarrow L_{q}(\mathcal{N})$ be completely positive. Then $T$ is completely order bounded and

$$
\|T\|_{c o b}=\|T\|=\sup \left\{\|T(x)\|_{q} \mid x \in L_{p}(\mathcal{M})_{+},\|x\|_{p} \leq 1\right\} .
$$

Here, $\|T\|$ means the usual operator norm of $T$ as a bounded operator on a normed vector space.

Proof Let $\Lambda=\sup \left\{\|T(x)\|_{q} \mid x \in L_{p}(\mathcal{M})_{+},\|x\|_{p} \leq 1\right\}$. By Theorem 3.6, we have 


$$
\sup \left\{\|T(x)\|_{q} \mid x \in L_{p}(\mathcal{M})_{+},\|x\|_{p} \leq 1\right\} \leq\|T\| \leq\|T\|_{c o b} .
$$

For the opposite inequality, let $n \in \mathbb{N}, x \in L_{p}(\mathcal{M}) \otimes M_{n}$ with $\|x\|_{p, n} \leq 1$. By Theorem 3.4, there exist $f, g \in L_{p}(\mathcal{M})_{+}$such that

$$
\|f\|_{p}=\|g\|_{p}=\|x\|_{p, n} \text { and }\left[\begin{array}{cc}
f \otimes 1_{n} & x \\
x^{*} & g \otimes 1_{n}
\end{array}\right] \geq 0 .
$$

Since $T$ is completely positive, we have $T_{n}\left(x^{*}\right)=T_{n}(x)^{*}$ and

$$
\left[\begin{array}{cc}
T(f) \otimes 1_{n} & T_{n}(x) \\
T_{n}(x)^{*} & T(g) \otimes 1_{n}
\end{array}\right] \geq 0
$$

Hence,

$$
\left\|T_{n}(x)\right\|_{q, n} \leq \frac{1}{2}\left(\|T(f)\|_{q}+\|T(g)\|_{q}\right) \leq \Lambda\|x\|_{p, n} \leq \Lambda .
$$

Theorem 4.3 Let $1 \leq p, q \leq \infty, \mathcal{M}$ and $\mathcal{N}$ be von Neumann algebras, and $T: L_{p}(\mathcal{M}) \rightarrow L_{q}(\mathcal{N})$ be a decomposable map. Then $T$ is completely order bounded and $\|T\|_{c o b} \leq\|T\|_{\text {dec }}$.

Proof For $\varepsilon>0$ there exist completely positive maps $S_{1}, S_{2}: L_{p}(\mathcal{M}) \rightarrow L_{q}(\mathcal{N})$ such that

$$
\Phi: L_{p}(\mathcal{M}) \otimes M_{2} \rightarrow L_{q}(\mathcal{N}) \otimes M_{2},\left[\begin{array}{ll}
x_{11} & x_{12} \\
x_{21} & x_{22}
\end{array}\right] \mapsto\left[\begin{array}{ll}
S_{1}\left(x_{11}\right) & T\left(x_{12}\right) \\
T\left(x_{21}^{*}\right)^{*} & S_{2}\left(x_{22}\right)
\end{array}\right]
$$

is completely positive and $\left\|S_{1}\right\|,\left\|S_{2}\right\| \leq\|T\|_{\text {dec }}+\varepsilon$. By Theorem 3.4, for $n \in \mathbb{N}, x=\left[x_{i j}\right] \in L_{p}\left(\mathcal{M} \otimes M_{n}\right.$, there exist $f, g \in L_{p}(\mathcal{M})_{+},\|f\|_{p}=\|g\|_{p}=\|x\|_{p, n}$ such that $a=\left[\begin{array}{cc}f \otimes 1_{n} & x \\ x^{*} & g \otimes 1_{n}\end{array}\right] \geq 0$. We multiply $a$ from left and right with a suitable permutation matrix to get $b=\left[b_{i j}\right]$ with $b_{i j}=\left[\begin{array}{cc}f & x_{i j} \\ x_{j i}^{*} & g\end{array}\right]$ for $i, j=1, \ldots, n$. Then $b$ is positive too, so $\Phi_{n}(b)$ is positive and $\Phi\left(b_{i j}\right)=\left[\begin{array}{cc}S_{1}(f) & T\left(x_{i j}\right) \\ T\left(x_{j i}\right)^{*} & S_{2}(g)\end{array}\right]$ for $i, j=1, \ldots, n$. Again, we multiply $\Phi_{n}(b)$ from left and right with a permutation matrix and get $\left[\begin{array}{cc}S_{1}(f) \otimes 1_{n} & T_{n}(x) \\ T_{n}(x)^{*} & S_{2}(g) \otimes 1_{n}\end{array}\right] \geq 0$. Thus

$$
\left\|T_{n}(x)\right\|_{q, n} \leq \frac{1}{2}\left(\left\|S_{1}(f)\right\|_{q}+\left\|S_{2}(g)\right\|_{q}\right) \leq\left(\|T\|_{d e c}+\varepsilon\right)\|x\|_{p, n} .
$$

Since $\varepsilon$ was arbitrary, the proof is finished.

Next, we show that the composition of complete order bounded maps is completely order bounded. 
Theorem 4.4 Let $1 \leq p_{1}, p_{2}, p_{3} \leq \infty, \mathcal{M}_{1}, \mathcal{M}_{2}, \mathcal{M}_{3}$ be von Neumann algebras, and $T_{1}: L_{p_{1}}\left(\mathcal{M}_{1}\right) \rightarrow L_{p_{2}}\left(\mathcal{M}_{2}\right), T_{2}: L_{p_{2}}\left(\mathcal{M}_{2}\right) \rightarrow L_{p_{3}}\left(\mathcal{M}_{3}\right)$ be completely order bounded maps. Then the composition $T_{2} \circ T_{1}$ is completely order bounded and

$$
\left\|T_{2} \circ T_{1}\right\|_{c o b} \leq\left\|T_{2}\right\|_{c o b}\left\|T_{1}\right\|_{c o b} \text {. }
$$

Proof Let $n \in \mathbb{N}$ and $x \in L_{p_{1}}\left(\mathcal{M}_{1}\right) \otimes M_{n}$ such that $\|x\|_{p_{1}, n} \leq 1$. Then

$$
\left.\left\|T_{2, n}\left(T_{1, n}(x)\right)\right\|_{p_{3}, n} \leq\left\|T_{2}\right\|_{c o b} \| T_{1, n}(x)\right)\left\|_{p_{2}, n} \leq\right\| T_{2}\left\|_{c o b}\right\| T_{1} \|_{c o b}
$$

The next theorem justifies the name completely order bounded: Completely order bounded maps map order intervals into order intervals uniformly over all matrix levels.

Theorem 4.5 Let $1 \leq p, q \leq \infty, T: L_{p}(\mathcal{M}) \rightarrow L_{q}(\mathcal{N})$ be completely order bounded, and $f_{1}, f_{2} \in L_{p}(\mathcal{M})_{+}$. Then there exist $g_{1}, g_{2} \in L_{q}(\mathcal{N})_{+}$such that $\left\|g_{1}\right\|_{q},\left\|g_{2}\right\|_{q} \leq \frac{1}{2}\|T\|_{c o b}\left(\left\|f_{1}\right\|_{p}+\left\|f_{2}\right\|_{p}\right)$ and for all $n \in \mathbb{N}, x \in L_{p}(\mathcal{M}) \otimes M_{n}$

$$
\left[\begin{array}{cc}
f_{1} \otimes 1_{n} & x \\
x^{*} & f_{2} \otimes 1_{n}
\end{array}\right] \geq 0 \text { implies }\left[\begin{array}{cc}
g_{1} \otimes 1_{n} & T_{n}(x) \\
T_{n}(x)^{*} & g_{2} \otimes 1_{n}
\end{array}\right] \geq 0
$$

Proof For $q>1, n \in \mathbb{N}, x \in L_{p}(\mathcal{M}) \otimes M_{n}$ with $\left[\begin{array}{cc}f_{1} \otimes 1_{n} & x \\ x^{*} & f_{2} \otimes 1_{n}\end{array}\right] \geq 0$, we put

$$
\begin{aligned}
K(x)=\{ & \left(g_{1}, g_{2}\right) \in\left(L_{q}(\mathcal{N})_{+}, L_{q}(\mathcal{N})_{+}\right) \mid \\
& {\left[\begin{array}{cc}
g_{1} \otimes 1_{n} & T_{n}(x) \\
T_{n}(x)^{*} & g_{2} \otimes 1_{n}
\end{array}\right] \geq 0, } \\
& \left.\left\|g_{1}\right\|_{q},\left\|g_{2}\right\|_{q} \leq \frac{1}{2}\|T\|_{c o b}\left(\left\|f_{1}\right\|_{p}+\left\|f_{2}\right\|_{p}\right)\right\} .
\end{aligned}
$$

For $q=1$ we define a similar set, but take pairs $\left(g_{1}, g_{2}\right) \in \mathcal{N}_{+}^{*} \times \mathcal{N}_{+}^{*}$ instead of $L_{1}(\mathcal{N})_{+} \times L_{1}(\mathcal{N})_{+}$. By Theorem 3.4, $K(x)$ is not empty. Further, $K(x)$ is weak*closed. This is proved similarly as in the proof of Theorem 3.4. By the BanachAlaoglu theorem, the unit ball of $L_{q}(\mathcal{N})$ is compact in the weak*-topology for $q>1$. The same holds for $\mathcal{N}^{*}$. Hence all sets $K(x)$ are compact in the weak *topology. The sets $K(x), x \in L_{p}(\mathcal{N}) \otimes M_{n}, n \in \mathbb{N}$, have the finite intersection property: For $k \in \mathbb{N}, n_{1}, \ldots, n_{k} \in \mathbb{N}$, and $x_{i} \in L_{p}(\mathcal{M}) \otimes M_{n_{i}}$ with

$$
\left[\begin{array}{cc}
f_{1} \otimes 1_{n_{i}} & x_{i} \\
x_{i}^{*} & f_{2} \otimes 1_{n_{i}}
\end{array}\right] \geq 0, i \in\{1, \ldots, k\},
$$

we put all $x_{i}$ in the diagonal matrix $x=\operatorname{diag}\left(x_{1}, \ldots, x_{k}\right)$ and set $n=\sum_{i=1}^{k} n_{i}$. Then 


$$
\left[\begin{array}{cc}
f_{1} \otimes 1_{n} & x \\
x^{*} & f_{2} \otimes 1_{n}
\end{array}\right] \geq 0 .
$$

By Theorem 3.4, there exist $g_{1}, g_{2} \in L_{q}(\mathcal{N})_{+}$such that

$$
\left[\begin{array}{cc}
g_{1} \otimes 1_{n} & T_{n}(x) \\
T_{n}(x)^{*} & g_{2} \otimes 1_{n}
\end{array}\right] \geq 0 \text { and }\left\|g_{1}\right\|,\left\|g_{2}\right\| \leq \frac{1}{2}\|T\|_{c o b}\left(\left\|f_{1}\right\|_{p}+\left\|f_{2}\right\|_{p}\right) .
$$

Hence, $\left(g_{1}, g_{2}\right) \in K\left(x_{i}\right)$ for all $i \in\{1, \ldots, k\}$. We conclude that

$$
\bigcap_{n \in \mathbb{N}, x \in L_{p}(\mathcal{M}) \otimes M_{n}} K(x) \neq \emptyset .
$$

We take a pair $\left(g_{1}, g_{2}\right)$ of this set. If $q>1$, this pair fulfills (4.2). If $q=1$ there is a central projection $z \in \mathcal{N}^{* *}$ which maps $\mathcal{N}^{*}$ to $L_{1}(\mathcal{N})$. Then the pair $\left(z g_{1}, z g_{2}\right)$ is in $L_{1}(\mathcal{N})_{+} \times L_{1}(\mathcal{N})_{+}$and fulfills (4.2).

Theorem 4.6 Let $1 \leq q \leq \infty$ and let $T: \mathcal{M} \rightarrow L_{q}(\mathcal{N})$ be completely order bounded. Then there exist $\left[f, g \in L_{q}(\mathcal{N})_{+}\right.$and a completely order bounded map $S: \mathcal{M} \rightarrow \mathcal{N}$ such that

$$
T(x)=f^{\frac{1}{2}} S(x) g^{\frac{1}{2}} \text { for all } x \in \mathcal{M}
$$

and

$$
\|f\|_{q},\|g\|_{q} \leq\|T\|_{c o b},\|S\|_{c o b} \leq 1
$$

Note that in case of a linear map from $\mathcal{M}$ to $\mathcal{N}$ completely order bounded is identical to completely bounded.

Proof Since the norm $\|\cdot\|_{\infty, n}$ is the usual operator norm on $\mathcal{M} \otimes M_{n}$, we get $\left[\begin{array}{cc}\mathbf{1} \otimes 1_{n} & x \\ x^{*} & \mathbf{1} \otimes 1_{n}\end{array}\right] \geq 0$ for all $n \in \mathbb{N}, x \in \mathcal{M} \otimes M_{n},\|x\|_{\infty, n} \leq 1$ where $\mathbf{1}$ is the unit in $\mathcal{M}$. Hence we can apply Theorem 4.5 with $f_{1}=f_{2}=\mathbf{1}$ and get $f, g \in L_{q}(\mathcal{N})$, such that $\|f\|_{q},\|g\|_{q} \leq\|T\|_{c o b}$ and

$$
\left[\begin{array}{cc}
f \otimes 1_{n} & T_{n}(x) \\
T_{n}(x)^{*} & g \otimes 1_{n}
\end{array}\right] \geq 0 \text { for all } n \in \mathbb{N}, x \in \mathcal{M} \otimes M_{n},\|x\|_{\infty, n} \leq 1 .
$$

Let $x \in \mathcal{M}$. We apply Theorem 2.3 with $n=1$ to $x$, and get a unique $y \in \mathcal{N},\|y\|_{\infty} \leq 1$ such that $T(x)=f^{\frac{1}{2}} y g^{\frac{1}{2}}$ and $\operatorname{supp}(f) \cdot y \cdot \operatorname{supp}(g)=y$. We put $S(x)=y$. Then $S$ is a map from $\mathcal{M}$ to $\mathcal{N}$. If $x, x_{1}, x_{2} \in \mathcal{M}, x=x_{1}+x_{2}$, then there are according to Theorem $2.3 y, y_{1}, y_{2} \in \mathcal{N}$ such that $T(x)=f^{\frac{1}{2}} y g^{\frac{1}{2}}, \operatorname{supp}(f) \cdot y$. $\operatorname{supp}(g)=y$ and $T\left(x_{i}\right)=f^{\frac{1}{2}} y_{i} g^{\frac{1}{2}}, \operatorname{supp}(f) \cdot y_{i} \cdot \operatorname{supp}(g)=y_{i}, i=1,2$. Then we get 


$$
\begin{array}{r}
f^{\frac{1}{2}} y g^{\frac{1}{2}}=T(x)=T\left(x_{1}\right)+T\left(x_{2}\right)=f^{\frac{1}{2}}\left(y_{1}+y_{2}\right)\left(g^{\frac{1}{2}}\right), \\
\operatorname{supp}(f) \cdot\left(y_{1}+y_{2}\right) \cdot \operatorname{supp}(g)=y_{1}+y_{2} .
\end{array}
$$

Hence we conclude from Lemma 2.2 that $y=y_{1}+y_{2}$ which means that $S$ is additive. Similarly, we show that $S(\lambda x)=\lambda S(x)$ for $\lambda \in \mathbb{C}, x \in \mathcal{M}$. Now for $n \in \mathbb{N}$, let $x \in \mathcal{M} \otimes M_{n}$ with $\|x\|_{\infty, n} \leq 1$. According to Theorem 2.3, there is $y \in \mathcal{N} \otimes M_{n}$ such that $\left(\operatorname{supp}(f) \otimes 1_{n}\right) \cdot y \cdot\left(\operatorname{supp}(g) \otimes 1_{n}\right)=y$ and $T_{n}(x)=\left(f^{\frac{1}{2}} \otimes 1_{n}\right) y\left(g^{\frac{1}{2}} \otimes 1_{n}\right)$. Then, we have

$$
\left(f^{\frac{1}{2}} \otimes 1_{n}\right) S_{n}(x)\left(g^{\frac{1}{2}} \otimes 1_{n}\right)=T_{n}(x)=\left(f^{\frac{1}{2}} \otimes 1_{n}\right) y\left(g^{\frac{1}{2}} \otimes 1_{n}\right) .
$$

Hence, we conclude that $S_{n}(x)=y$ which shows that $\left\|S_{n}(x)\right\|_{\infty, n} \leq 1$. So $\|S\|_{c o b} \leq 1$.

Theorem 4.7 Let $\mathcal{N}$ be injective, $\quad 1 \leq q \leq \infty$ and let $T: \mathcal{M} \rightarrow L_{q}(\mathcal{N})$ be completely order bounded. Then there exist linear maps $T_{i}: \mathcal{M} \rightarrow L_{q}(\mathcal{N})$ such that the map

$$
\begin{aligned}
\Phi: \mathcal{M} \otimes M_{2} \rightarrow L_{q}(\mathcal{N}) \otimes M_{2} \\
\left.\qquad \begin{array}{ll}
x_{11} & x_{12} \\
x_{21} & x_{22}
\end{array}\right] \mapsto\left[\begin{array}{ll}
T_{1}\left(x_{11}\right) & T\left(x_{12}\right) \\
T\left(x_{21}^{*}\right)^{*} & T_{2}\left(x_{22}\right)
\end{array}\right]
\end{aligned}
$$

is complete positive and $\left\|T_{1}\right\|,\left\|T_{2}\right\| \leq\|T\|_{c o b}$. Thus $T$ is decomposable and $\|T\|_{\text {dec }}=\|T\|_{c o b}$.

Proof By Theorem 4.6, there exist $f, g \in L_{q}(\mathcal{N})_{+},\|f\|_{q},\|g\|_{q} \leq\|T\|_{c o b}$ and $S$ : $\mathcal{M} \rightarrow \mathcal{N},\|S\|_{c o b} \leq 1$ such that

$$
T(x)=f^{\frac{1}{2}} S(x) g^{\frac{1}{2}}, \quad x \in \mathcal{M} .
$$

Since for a linear map from $\mathcal{M}$ to $\mathcal{N}$ completely bounded is the same as completely order bounded, we can apply [14], Theorem 4.5, and get linear maps $S_{1}, S_{2}: \mathcal{M} \rightarrow$ $\mathcal{N}$ such that $\left\|S_{i}\right\| \leq 1, i=1,2$, and the map

$$
\left[\begin{array}{ll}
x_{11} & x_{12} \\
x_{21} & x_{22}
\end{array}\right] \mapsto\left[\begin{array}{ll}
S_{1}\left(x_{11}\right) & S\left(x_{12}\right) \\
S\left(x_{21}^{*}\right)^{*} & S_{2}\left(x_{22}\right)
\end{array}\right]
$$

is completely positive. The linear maps $T_{i}: \mathcal{M} \rightarrow L_{q}(\mathcal{N})$ where $T_{1}(x)=$ $f^{\frac{1}{2}} S_{1}(x) f^{\frac{1}{2}}, T_{2}(x)=g^{\frac{1}{2}} S_{2}(x) g^{\frac{1}{2}}, x \in \mathcal{M}, \quad$ fulfill $\quad\left\|T_{1}\right\| \leq\|f\|_{q}\left\|S_{1}\right\| \leq\|T\|_{c o b}$, $\left\|T_{2}\right\| \leq\|g\|_{q}\left\|S_{2}\right\| \leq\|T\|_{c o b}$, and

$$
\mathcal{M} \otimes M_{2} \ni\left[\begin{array}{ll}
x_{11} & x_{12} \\
x_{21} & x_{22}
\end{array}\right] \mapsto\left[\begin{array}{ll}
T_{1}\left(x_{11}\right) & T\left(x_{12}\right) \\
T\left(x_{21}^{*}\right)^{*} & T_{2}\left(x_{22}\right)
\end{array}\right] \in L_{q}(\mathcal{N}) \otimes M_{2}
$$

is completely positive. Together with Theorem 4.3 we get $\|T\|_{d e c}=\|T\|_{c o b}$ 
Remark 4.8 Theorem 4.5 in [14] states the decomposition for self-adjoint maps. The proof of Proposition 1.3 in [5] shows that this is also true for maps which are not self-adjoint.

The next goal is to prove that completely order bounded maps from $L_{p}(\mathcal{M})$ to $L_{1}(\mathcal{N})$. are decomposable. This proof is divided into several steps.

Lemma 4.9 Let $k \in \mathbb{N}$ and $a \in L_{p}(\mathcal{M}) \otimes M_{2} \otimes M_{2 k}$ be self-adjoint. Then a can be written in the form

$$
a=\sum_{j=1}^{n} a_{j} \otimes \alpha_{j}
$$

where all $a_{j} \in L_{p}(\mathcal{M}) \otimes M_{2}$ and all $\alpha_{j} \in M_{2 k}$ are self-adjoint.

Proof We mimic the proof of [12], Lemma IV.4.4. If $a=\sum_{j=1}^{n} b_{j} \otimes \beta_{j}$ with $b_{j} \in$ $\mathcal{M} \otimes M_{2}$ and $\beta_{j} \in M_{24}$ for $j=1, \ldots, n$ and $a$ is self-adjoint then

$$
a=\frac{1}{4} \sum_{j=1}^{n}\left(\left(b_{j}+b_{j}^{*}\right) \otimes\left(\beta_{j}+\beta_{j}^{*}\right)-i\left(b_{j}-b_{j}^{*}\right) \otimes i\left(\beta_{j}-\beta_{j}^{*}\right)\right)
$$

Let $\operatorname{Tr}$ be the usual trace on $M_{2 k}$. We define the duality between $L_{1}\left(M_{k}\right) \otimes M_{2}$ and $M_{2 k}$ by $\langle a, b\rangle=\operatorname{Tr}(a b), a \in L_{1}\left(M_{k}\right) \otimes M_{2}, b \in M_{2 k}$. If $n \in \mathbb{N}, a=\left[a_{i j}\right], b=$ $\left[b_{i j}\right]$, where $a_{i j} \in L_{1}\left(M_{k}\right) \otimes M_{2}$ and $b_{i j} \in M_{k} \otimes M_{2}$ then $\left\langle\left[a_{i j}\right],\left[b_{i j}\right]\right\rangle=\sum_{i, j=1}^{2 k}\left\langle a_{i j}, b_{j i}\right\rangle$. We define the linear functional

$$
\begin{gathered}
\omega: L_{1}\left(M_{k}\right) \otimes M_{2} \otimes M_{2 k} \rightarrow \mathbb{C} \\
\sum_{i=1}^{n} a_{i} \otimes b_{i} \mapsto \sum_{i=1}^{n}\left\langle a_{i}, b_{i}^{t}\right\rangle
\end{gathered}
$$

where $b_{i}^{t}$ denotes the transposed matrix of $b_{i}$.

Lemma 4.10 Let $a \in\left(L_{1}\left(M_{k}\right) \otimes M_{2} \otimes M_{2 k}\right)_{+}$. Then $\omega(a) \geq 0$.

Proof Let $a^{\frac{1}{2}}=\sum_{i=1}^{n} a_{i} \otimes \alpha_{i}$. Then $a^{\frac{1}{2}}$ is self-adjoint, $a=\sum_{i, j=1}^{n} a_{i}^{*} a_{j} \otimes \alpha_{i}^{*} \alpha_{j}$, and

$$
\begin{aligned}
\omega(a) & =\sum_{i, j=1}^{n} \omega\left(a_{i}^{*} a_{j} \otimes \alpha_{i}^{*} \alpha_{j}\right)=\sum_{i, j=1}^{n}\left\langle a_{i}^{*} a_{j}, \alpha_{j}^{t} \alpha_{i}^{* t}\right\rangle \\
& =\left\langle\left[a_{i}^{*} a_{j}\right],\left[\alpha_{i}^{t} \alpha_{j}^{* t}\right]\right\rangle
\end{aligned}
$$

Since $\left[a_{i}^{*} a_{j}\right]$ and $\left[\alpha_{i}^{t} \alpha_{j}^{* t}\right]$ are positive matrices, the last expression is positive by (2.3).

For a linear map $T: L_{p}(\mathcal{M}) \rightarrow L_{1}\left(M_{k}\right)$ we define 


$$
\begin{gathered}
\tilde{T}: L_{p}(\mathcal{M}) \otimes M_{2} \rightarrow L_{1}\left(M_{k}\right) \otimes M_{2} \\
{\left[\begin{array}{ll}
x_{11} & x_{12} \\
x_{21} & x_{22}
\end{array}\right] \mapsto\left[\begin{array}{cc}
0 & T\left(x_{12}\right) \\
T\left(x_{21}^{*}\right)^{*} & 0
\end{array}\right] .}
\end{gathered}
$$

We define the linear functional

$$
\begin{aligned}
\varphi_{T}: L_{p}(\mathcal{M}) \otimes M_{2} \otimes M_{2 k} \rightarrow \mathbb{C} \\
x \mapsto \omega \circ \tilde{T}_{2 k}(x) .
\end{aligned}
$$

Lemma 4.11 Let $T: L_{p}(\mathcal{M}) \rightarrow L_{1}\left(M_{k}\right)$ be completely order bounded and $\varphi_{T}$ as in (4.5). Let $a, b \in\left(L_{p}(\mathcal{M}) \otimes M_{2} \otimes M_{2 k}\right)_{+}, f, g \in L_{p}(\mathcal{M})_{+}$such that

$$
0 \leq a+b \leq f \otimes \varepsilon_{11} \otimes 1_{2 k}+g \otimes \varepsilon_{22} \otimes 1_{2 k} .
$$

Then

$$
\|T\|_{c o b}\left(\|f\|_{p}+\|g\|_{p}\right)+\varphi_{T}(a-b) \geq 0
$$

Here $\varepsilon_{i j}, i, j=1,2$ are the $2 \times 2$ matrices defined in (3.2).

Proof Since $a-b$ is self-adjoint, by Lemma 4.9 , we can write

$$
a-b=\sum_{i=1}^{n} a_{i} \otimes \alpha_{i}
$$

where all $a_{i} \in L_{p}(\mathcal{M}) \otimes M_{2}$ and all $\alpha_{i} \in M_{2 k}$ are self-adjoint. For $i \in\{1, \ldots, n\}$ let

$$
a_{i}=\left[\begin{array}{ll}
a_{i, 11} & a_{i, 12} \\
a_{i, 21} & a_{i, 22}
\end{array}\right], \alpha_{i}=\left[\begin{array}{cc}
\alpha_{i, 11} & \alpha_{i, 12} \\
\alpha_{i, 21} & \alpha_{i, 22}
\end{array}\right],
$$

where $a_{i, s t} \in L_{p}(\mathcal{M})$ and $\alpha_{i, s t} \in M_{k}$ for $s, t=1$, 2. Since $a_{i}$ and $\alpha_{i}$ are self-adjoint for all $i$, we have $a_{i, 21}^{*}=a_{i, 12}, \alpha_{i, 21}^{*}=\alpha_{i, 12}$, and $\left\langle T\left(a_{i, 21}^{*}\right)^{*}, \alpha_{i, 21}^{t}\right\rangle=\left\langle T\left(a_{i, 12}\right)^{*}, \alpha_{i, 12}^{* t}\right\rangle=$ $\overline{\left\langle T\left(a_{i, 12}\right), \alpha_{i, 12}^{t}\right\rangle}$ for all $i \in\{1, \ldots, n\}$. Therefore

$$
\begin{aligned}
\varphi_{T}(a-b) & =\sum_{i=1}^{n} \varphi_{T}\left(a_{i} \otimes \alpha_{i}\right) \\
& =\sum_{i=1}^{n}\left\langle\left[\begin{array}{cc}
0 & T\left(a_{i, 12}\right) \\
T\left(a_{i, 21}^{*}\right)^{*} & 0
\end{array}\right],\left[\begin{array}{cc}
\alpha_{i, 11}^{t} & \alpha_{i, 21}^{t} \\
\alpha_{i, 12}^{t} & \alpha_{i, 22}^{t}
\end{array}\right]\right\rangle \\
& =\sum_{i=1}^{n}\left(\left\langle T\left(a_{i, 12}\right), \alpha_{i, 12}^{t}\right\rangle+\left\langle T\left(a_{i, 12}\right)^{*}, \alpha_{i, 12}^{* t}\right\rangle\right) .
\end{aligned}
$$

By Proposition 2.1, we have 


$$
\left[\begin{array}{cc}
\left(f \otimes \varepsilon_{11}+g \otimes \varepsilon_{22}\right) \otimes 1_{2 k} & a-b \\
a-b & \left(f \otimes \varepsilon_{11}+g \otimes \varepsilon_{22}\right) \otimes 1_{2 k}
\end{array}\right] \geq 0 .
$$

We multiply the matrix in (4.7) from left with the matrix $\gamma$ and from right with the transposed matrix $\gamma^{t}$, where $\gamma$ is the $8 k \times 4 k$ matrix $\left[\begin{array}{l}\varepsilon_{11} \otimes 1_{2 k} \\ \varepsilon_{22} \otimes 1_{2 k}\end{array}\right]$ and get

$$
\begin{aligned}
0 \leq & \varepsilon_{11}\left(f \otimes \varepsilon_{11}+g \otimes \varepsilon_{22}\right) \varepsilon_{11} \otimes 1_{2 k}+\left(\varepsilon_{11} \otimes 1_{2 k}\right)(a-b)\left(\varepsilon_{22} \otimes 1_{2 k}\right) \\
& +\left(\varepsilon_{22} \otimes 1_{2 k}\right)(a-b)\left(\varepsilon_{11} \otimes 1_{2 k}\right)+\varepsilon_{22}\left(f \otimes \varepsilon_{11}+g \otimes \varepsilon_{22}\right) \varepsilon_{22} \otimes 1_{2 k} \\
= & f \otimes \varepsilon_{11} \otimes 1_{2 k}+\sum_{i=1}^{n} a_{i, 12} \otimes \varepsilon_{12} \otimes \alpha_{i} \\
& +\sum_{i=1}^{n} a_{i, 21} \otimes \varepsilon_{21} \otimes \alpha_{i}+g \otimes \varepsilon_{22} \otimes 1_{2 k} .
\end{aligned}
$$

This inequality can be written as $\left\|\sum_{i=1}^{n} a_{i, 12} \otimes \alpha_{i}\right\|_{p, 2 k} \leq \frac{1}{2}\left(\|f\|_{p}+\|g\|_{p}\right)$. Hence $\left\|T\left(\sum_{i=1}^{n} a_{i, 12} \otimes \alpha_{i}\right)\right\|_{1,2 k} \leq \frac{1}{2}\|T\|_{c o b}\left(\|f\|_{p}+\|g\|_{p}\right)$. By Theorem 3.4, there exist $f_{1}, g_{1} \in L_{1}\left(M_{k}\right)_{+}$such that $\left\|f_{1}\right\|_{1}=\left\|g_{1}\right\|_{1} \leq \frac{1}{2}\|T\|_{c o b}\left(\|f\|_{p}+\|g\|_{p}\right)$ and

$$
\left[\begin{array}{cc}
f_{1} \otimes 1_{2 k} & \sum_{i=1}^{n} T\left(a_{i, 12}\right) \otimes \alpha_{i} \\
\sum_{i=1}^{n} T\left(a_{i, 12}\right)^{*} \otimes \alpha_{i} & g_{1} \otimes 1_{2 k}
\end{array}\right] \geq 0 .
$$

We apply $\omega$ to (4.9) and get

$$
\begin{aligned}
0 \leq & \left\langle f_{1} \otimes \varepsilon_{11}, 1_{2 k}\right\rangle+\left\langle g_{1} \otimes \varepsilon_{22}, 1_{2 k}\right\rangle \\
& +\sum_{i=1}^{n}\left(\left\langle T\left(a_{i, 12}\right) \otimes \varepsilon_{12}, \alpha_{i}^{t}\right\rangle+\left\langle T\left(a_{i, 12}\right)^{*} \otimes \varepsilon_{21}, \alpha_{i}^{t}\right\rangle\right) \\
= & \left\|f_{1}\right\|_{1}+\left\|g_{1}\right\|_{1}+\sum_{i=1}^{n}\left(\left\langle T\left(a_{i, 12}\right), \alpha_{i, 12}^{t}\right\rangle+\left\langle T\left(a_{i, 12}\right)^{*}, \alpha_{i, 12}^{* t}\right\rangle\right) \\
= & \left\|f_{1}\right\|_{1}+\left\|g_{1}\right\|_{1}+\varphi_{T}(a-b) .
\end{aligned}
$$

Since $\left\|f_{1}\right\|_{1}+\left\|g_{1}\right\|_{1} \leq\|T\|_{c o b}\left(\|f\|_{p}+\|g\|_{p}\right)$, the proof is finished.

Lemma 4.12 Let $x \in\left(L_{p}(\mathcal{M}) \otimes M_{2} \otimes M_{n}\right)_{+}$and $y \in\left(M_{k} \otimes M_{2} \otimes M_{n}\right)_{+}$where $x=\left[x_{i j}\right]$ with $\quad x_{i j} \in L_{p}\left(\mathcal{M} \otimes M_{2}\right)$ and $y=\left[y_{i j}\right]$ with $y_{i j} \in M_{k} \otimes M_{2}$ for $i, j \in$ $\{1, \ldots, n\}$ Then

$$
\sum_{i, j=1}^{n} x_{i j} \otimes y_{j i}^{t} \geq 0
$$

Proof By Lemma 2.3, there is $f \in L_{p}(\mathcal{M})_{+}$and $a \in\left(\mathcal{M} \otimes M_{2} \otimes M_{n}\right)_{+}$such that $x=\left(f^{\frac{1}{2}} \otimes 1_{2 n}\right) a\left(f^{\frac{1}{2}} \otimes 1_{2 n}\right)$. By [12], Lemma IV.3.1, $a$ can be written as a finite sum 
of matrices of the form $\left[a_{i}^{*} a_{j}\right]$ where $a_{1}, \ldots, a_{n} \in \mathcal{M} \otimes M_{2}$. Similarly, $y$ can be written as a finite sum of matrices of the form $\left[y_{i}^{*} y_{j}\right]$ where $y_{1}, \ldots, y_{n} \in M_{k} \otimes M_{2}$. Thus $\sum_{i, j=1}^{n} x_{i j} \otimes y_{i j}^{t}$ is a finite sum of elements of the form $\sum_{i, j=1}^{n}\left(f^{\frac{1}{2}} \otimes 1_{2}\right) a_{i}^{*} a_{j}\left(f^{\frac{1}{2}} \otimes 1_{2}\right) \otimes\left(y_{i}^{*} y_{j}\right)^{t}$. Then we have

$$
\begin{aligned}
& \sum_{i, j=1}^{n}\left(f^{\frac{1}{2}} \otimes 1_{2}\right) a_{i}^{*} a_{j}\left(f^{\frac{1}{2}} \otimes 1_{2}\right) \otimes\left(y_{j}^{*} y_{i}\right)^{t} \\
= & \sum_{i, j=1}^{n}\left(f^{\frac{1}{2}} \otimes 1_{2}\right) a_{i}^{*} a_{j}\left(f^{\frac{1}{2}} \otimes 1_{2}\right) \otimes y_{i}^{t} y_{j}^{* t} \\
= & \left(\sum_{i=1}^{n} a_{i}\left(f^{\frac{1}{2}} \otimes 1_{2}\right) \otimes y_{i}^{* t}\right)^{*}\left(\sum_{j=1}^{n} a_{j}\left(f^{\frac{1}{2}} \otimes 1_{2}\right) \otimes y_{j}^{* t}\right) \geq 0
\end{aligned}
$$

Proposition 4.13 Let $T: L_{p}(\mathcal{M}) \rightarrow L_{1}\left(M_{k}\right)$ be completely order bounded and let $\tilde{T}$ be as in (4.4). Then there is a linear map $S: L_{p}(\mathcal{M}) \otimes M_{2} \rightarrow L_{1}\left(M_{k}\right) \otimes M_{2}$ such that $S \pm \tilde{T}$ are completely positive and

$$
\begin{aligned}
& 0 \leq\left\langle S\left(\left[\begin{array}{ll}
c & 0 \\
0 & 0
\end{array}\right]\right),\left[\begin{array}{ll}
y & 0 \\
0 & 0
\end{array}\right]\right\rangle \leq\|T\|_{c o b}\|c\|_{p}\|y\|_{\infty} \\
& 0 \leq\left\langle S\left(\left[\begin{array}{ll}
0 & 0 \\
0 & c
\end{array}\right]\right),\left[\begin{array}{ll}
0 & 0 \\
0 & y
\end{array}\right]\right\rangle \leq\|T\|_{c o b}\|c\|_{p}\|y\|_{\infty}
\end{aligned}
$$

for all $c \in L_{p}(\mathcal{M})_{+}$and all $y \in M_{k+}$.

Proof Let $\left(L_{p}(\mathcal{M}) \otimes M_{2} \otimes M_{2 k}\right)_{h}$ denote the self-adjoint part of $L_{p}(\mathcal{M}) \otimes M_{2} \otimes$ $M_{2 k}$ and let $\varphi_{T}$ be the linear functional defined by (4.5). For $x \in\left(L_{p}(\mathcal{M}) \otimes M_{2} \otimes\right.$ $\left.M_{2 k}\right)_{h}$ we define

$$
\begin{gathered}
\theta(x)=\inf \left\{\|T\|_{c o b}\left(\|f\|_{p}+\|g\|_{p}\right)+\varphi_{T}(a-b) \mid\right. \\
a, b \in\left(L_{p}(\mathcal{M}) \otimes M_{2} \otimes M_{2 k}\right)_{+}, f, g \in L_{p}(\mathcal{M})_{+}, \\
\left.x+a+b \leq f \otimes \varepsilon_{11} \otimes 1_{2 k}+g \otimes \varepsilon_{22} \otimes 1_{2 k}\right\} .
\end{gathered}
$$

By Lemma 2.4, the set on the right side of (4.11) is not empty, so $\theta$ is well defined. We will show that $\theta$ is sublinear. To do this, let $x_{1}, x_{2} \in\left(L_{p}(\mathcal{M}) \otimes M_{2} \otimes M_{2 k}\right)_{h}$ and $\varepsilon>0$. Then there exist $a_{1}, b_{1}, a_{2}, b_{2} \in\left(L_{p}(\mathcal{M}) \otimes M_{2} \otimes M_{2 k}\right)_{+}$and $f_{1}, g_{1}, f_{2}, g_{2} \in$ $L_{p}(\mathcal{M})_{+}$such that 


$$
\begin{aligned}
& x_{1}+a_{1}+b_{1} \leq f_{1} \otimes \varepsilon_{11} \otimes 1_{2 k}+g_{1} \otimes \varepsilon_{22} \otimes 1_{2 k}, \\
& x_{2}+a_{2}+b_{2} \leq f_{2} \otimes \varepsilon_{11} \otimes 1_{2 k}+g_{2} \otimes \varepsilon_{22} \otimes 1_{2 k}, \\
& \theta\left(x_{1}\right) \geq\|T\|_{c o b}\left(\left\|f_{1}\right\|_{p}+\left\|g_{1}\right\|_{p}\right)+\varphi_{T}\left(a_{1}-b_{1}\right)-\varepsilon, \\
& \theta\left(x_{2}\right) \geq\|T\|_{c o b}\left(\left\|f_{2}\right\|_{p}+\left\|g_{2}\right\|_{p}\right)+\varphi_{T}\left(a_{2}-b_{2}\right)-\varepsilon .
\end{aligned}
$$

Then we get

$$
x_{1}+x_{2}+a_{1}+a_{2}+b_{1}+b_{2} \leq\left(f_{1}+f_{2}\right) \otimes \varepsilon_{11} \otimes 1_{2 k}+\left(g_{1}+g_{2}\right) \otimes \varepsilon_{22} \otimes 1_{2 k} .
$$

This implies

$$
\theta\left(x_{1}+x_{2}\right) \leq \theta\left(x_{1}\right)+\theta\left(x_{2}\right)-2 \varepsilon .
$$

Since $\varepsilon$ was arbitrary, $\theta$ is sub-additive. Similarly, we show for $0<\lambda \in \mathbb{R}$ and $x \in\left(L_{p}(\mathcal{M}) \otimes M_{2} \otimes M_{2 k}\right)_{h}$ that

$$
\theta(\lambda x) \leq \lambda \theta(x)
$$

and hence

$$
\lambda \theta(x)=\lambda \theta\left(\frac{1}{\lambda} \lambda x\right) \leq \lambda \frac{1}{\lambda} \theta(\lambda x)=\theta(\lambda x) .
$$

It remains to show that $\theta(0)=0$. If we put $x=a=b=0, f=g=0$ in (4.11), we get $\theta(0) \leq 0$. Lemma 4.11 states that every element in the set on the right side of (4.11) is not negative for $x=0$. Hence $\theta(0)=0$. By the Hahn-Banach theorem there is a real-linear functional

$$
\psi:\left(L_{p}(\mathcal{M}) \otimes M_{2} \otimes M_{2 k}\right)_{h} \rightarrow \mathbb{R}
$$

such that $\psi(x) \leq \theta(x)$ for all $x \in\left(L_{p}(\mathcal{M}) \otimes M_{2} \otimes M_{2 k}\right)_{h}$. Now we can extend $\psi$ to a complex linear functional on $L_{p}(\mathcal{M}) \otimes M_{2} \otimes M_{2 k}$ by putting $\psi(x)=\frac{1}{2} \psi\left(x+x^{*}\right)+$ $\frac{1}{2} \mathrm{i} \psi\left(\mathrm{i} x^{*}-\mathrm{i} x\right)$ for $x \in L_{p}(\mathcal{M}) \otimes M_{2} \otimes M_{2 k}$. For $c \in\left(L_{p}(\mathcal{M}) \otimes M_{2}\right)_{+},\|c\|_{p, 2} \leq 1$, by Theorem 3.4, there exists $f \in L_{p}(\mathcal{M})_{+}$such that

$$
f \otimes 1_{2}-c \geq 0 \text { and }\|f\|_{p} \leq 1 .
$$

For $y \in\left(M_{2 k}\right)_{+}, 0 \leq y \leq 1_{2 k}$, we put $x=c \otimes y^{t}, a=b=0$, apply (4.11), and get

$$
\psi\left(c \otimes y^{t}\right) \leq \theta\left(c \otimes y^{t}\right) \leq 2\|T\|_{c o b}\|f\|_{p} \leq 2\|T\|_{c o b} .
$$

Then we put $x=-c \otimes y^{t}, a=c \otimes y^{t}, b=0, f=g=0$, apply (4.11), and get

$$
\psi\left(-c \otimes y^{t}\right) \leq \theta\left(-c \otimes y^{t}\right) \leq \varphi_{T}\left(c \otimes y^{t}\right)
$$

For $x=-c \otimes y^{t}, a=0, b=c \otimes y^{t}, f=g=0$, we apply (4.11) and (4.12) and get 


$$
\psi\left(-c \otimes y^{t}\right) \leq \theta\left(-c \otimes y^{t}\right) \leq-\varphi_{T}\left(c \otimes y^{t}\right)
$$

We combine (4.13), (4.14), (4.15), and get

$$
0 \leq \psi\left(c \otimes y^{t}\right) \leq 2\|T\|_{c o b} .
$$

By Theorem 2.3 and [2], Proposition II.3.1.2, we can write $c \in L_{p}(\mathcal{M}) \otimes$ $M_{2},\|c\|_{p, 2} \leq 1$ as sum $c=c_{1}-c_{2}+\mathrm{i}\left(c_{3}-c_{4}\right)$ where $c_{i} \geq 0$ and $\left\|c_{i}\right\|_{p, 2} \leq 1$ for $i=1, \ldots, 4$. A similar decomposition holds for $y \in M_{2 k}$. Thus the bilinear map

$$
B: L_{p}(\mathcal{M}) \otimes M_{2} \times M_{2 k} \rightarrow \mathbb{C}, B(c, y)=\psi\left(c \otimes y^{t}\right)
$$

is bounded. Hence there is a linear map $S: L_{p}(\mathcal{M}) \otimes M_{2} \rightarrow L_{1}\left(M_{k}\right) \otimes M_{2}$ such that $\langle S(c), y\rangle=\psi\left(c \otimes y^{t}\right)$. Next we show that $S \pm \tilde{T}$ are completely positive. By (2.3), it suffices to show that for $n \in \mathbb{N}, x \in\left(L_{p}(\mathcal{M}) \otimes M_{2} \otimes M_{n}\right)_{+}, y \in\left(M_{k} \otimes M_{2} \otimes M_{n}\right)_{+}$, where $x=\left[x_{i j}\right]$ with $x_{i j} \in L_{p}(\mathcal{M}) \otimes M_{2}$ and $y=\left[y_{i j}\right] \in M_{k} \otimes M_{2}$ the expression $\left\langle S_{n}(x) \pm \tilde{T}_{n}(x), y\right\rangle$ is positive. Now we have

$$
\begin{aligned}
\left\langle S_{n}(x) \pm \tilde{T}_{n}(x), y\right\rangle & =\sum_{i, j=1}^{n}\left\langle S\left(x_{i j}\right) \pm \tilde{T}\left(x_{i j}\right), y_{j i}\right\rangle \\
& =\psi\left(\sum_{i, j=1}^{n} x_{i j} \otimes y_{j i}^{t}\right) \pm \varphi_{T}\left(\sum_{i, j=1}^{n} x_{i j} \otimes y_{j i}^{t}\right) .
\end{aligned}
$$

By Lemma 4.12, $\sum_{i, j=1}^{n} x_{i j} \otimes y_{j i}^{t}$ is positive. We put $x=-\sum_{i, j=1}^{n} x_{i j} \otimes y_{j i}^{t}, a=$ $-x, b=0, f=g=0$ in (4.11) and get

$$
-\psi\left(\sum_{i, j=1}^{n} x_{i j} \otimes y_{j i}^{t}\right) \leq \varphi_{T}\left(\sum_{i, j=1}^{n} x_{i j} \otimes y_{j i}^{t}\right) .
$$

Similarly, we put $x=\sum_{i, j=1}^{n} x_{i j} \otimes y_{j i}^{t}, a=0, b=-x, f=g=0$ and get

$$
-\psi\left(\sum_{i, j=1}^{n} x_{i j} \otimes y_{j i}^{t}\right) \leq-\varphi_{T}\left(\sum_{i, j=1}^{n} x_{i j} \otimes y_{j i}^{t}\right) .
$$

The combination of (4.16), (4.17), and (4.18) gives

$$
\left\langle S_{n}(x) \pm \tilde{T}_{n}(x), y\right\rangle \geq 0 .
$$

Now let $\quad c \in L_{p}(\mathcal{M})_{+} \quad$ and $\quad y \in M_{k+}$. We put $x=c \otimes \varepsilon_{11} \otimes y^{t} \otimes \varepsilon_{11}, a=b=0, f=\|y\|_{\infty} \cdot c, g=0$. Then (4.11) gives

$$
0 \leq\left\langle S\left(\left[\begin{array}{ll}
c & 0 \\
0 & 0
\end{array}\right]\right),\left[\begin{array}{ll}
y & 0 \\
0 & 0
\end{array}\right]\right\rangle=\psi(x) \leq \theta(x) \leq\|T\|_{c o b}\|c\|_{p}\|y\|_{\infty} .
$$

The second part of (4.10) is shown similarly. 
Proposition 4.14 Let $k \in \mathbb{N}$ and $T: L_{p}(\mathcal{M}) \rightarrow L_{1}\left(M_{k}\right)$ be completely order bounded. Then there are linear maps $S_{1}, S_{2}: L_{p}(\mathcal{M}) \rightarrow L_{1}\left(M_{k}\right)$ such that the map

$$
\begin{gathered}
\Phi: L_{p}(\mathcal{M}) \otimes M_{2} \rightarrow L_{1}\left(M_{k}\right) \otimes M_{2} \\
{\left[\begin{array}{ll}
x_{11} & x_{12} \\
x_{21} & x_{22}
\end{array}\right] \mapsto\left[\begin{array}{ll}
S_{1}\left(x_{11}\right) & T\left(x_{12}\right) \\
T\left(x_{21}^{*}\right)^{*} & S_{2}\left(x_{22}\right)
\end{array}\right]}
\end{gathered}
$$

is completely positive and $\left\|S_{1}\right\|,\left\|S_{2}\right\| \leq\|T\|_{\text {cob }}$.

Proof Let $\tilde{T}$ be as in (4.4). By Proposition 4.13, there is a linear map $S$ : $L_{p}(\mathcal{M}) \otimes M_{2} \rightarrow L_{1}\left(M_{k}\right) \otimes M_{2}$ such that $S \pm \tilde{T}$ are completely positive and for $c \in L_{p}(\mathcal{M})_{+}, y \in M_{k+}$

$$
\begin{aligned}
& 0 \leq\left\langle S\left(\left[\begin{array}{ll}
c & 0 \\
0 & 0
\end{array}\right]\right),\left[\begin{array}{ll}
y & 0 \\
0 & 0
\end{array}\right]\right\rangle \leq\|T\|_{c o b}\|c\|_{p}\|y\|_{\infty} \\
& 0 \leq\left\langle S\left(\left[\begin{array}{ll}
0 & 0 \\
0 & c
\end{array}\right]\right),\left[\begin{array}{ll}
0 & 0 \\
0 & y
\end{array}\right]\right\rangle \leq\|T\|_{c o b}\|c\|_{p}\|y\|_{\infty} .
\end{aligned}
$$

The next steps are quite similar to the proof of [1], Proposition 3.18. Let $\alpha$ be the scalar $1 \times 2$ matrix [10] and $\beta$ be the scalar $1 \times 2$ matrix [01], and let $\alpha^{*}, \beta^{*}$ be the adjoined matrices. We put

$$
S_{1}: L_{p}(\mathcal{M}) \rightarrow L_{1}\left(M_{k}\right), a \mapsto \alpha S\left(\left[\begin{array}{ll}
a & 0 \\
0 & 0
\end{array}\right]\right) \alpha^{*}
$$

and

$$
S_{2}: L_{p}(\mathcal{M}) \rightarrow L_{1}\left(M_{k}\right), a \mapsto \beta S\left(\left[\begin{array}{ll}
0 & 0 \\
0 & a
\end{array}\right]\right) \beta^{*}
$$

Since symmetric multiplication with a matrix and its adjoint is completely positive, the maps $S_{1}$ and $S_{2}$ are completely positive. For $a \in L_{p}(\mathcal{M})_{+}$, we have

$$
\begin{aligned}
\left\|S_{1}(a)\right\|_{1} & =\left\langle S_{1}(a), 1_{k}\right\rangle=\left\langle\alpha S\left(\left[\begin{array}{ll}
a & 0 \\
0 & 0
\end{array}\right]\right) \alpha^{*}, 1_{k}\right\rangle \\
& =\left\langle S\left(\left[\begin{array}{ll}
a & 0 \\
0 & 0
\end{array}\right]\right),\left[\begin{array}{ll}
1 & 0 \\
0 & 0
\end{array}\right]\right\rangle \leq\|T\|_{c o b}\|a\|_{p} .
\end{aligned}
$$

Hence $\left\|S_{1}\right\| \leq\|T\|_{c o b}$. Similarly, $\left\|S_{2}\right\| \leq\|T\|_{c o b}$. Let $\gamma_{1}$ be the scalar $2 \times 4$ matrix $\left[\begin{array}{llll}1 & 0 & 0 & 0 \\ 0 & 0 & 0 & 1\end{array}\right]$. For $a=\left[a_{i j}\right] \in L_{p}(\mathcal{M}) \otimes M_{2}$ we have 


$$
\gamma_{1}^{*}\left[\begin{array}{ll}
a_{11} & a_{12} \\
a_{21} & a_{22}
\end{array}\right] \gamma_{1}=\left[\begin{array}{cccc}
a_{11} & 0 & 0 & a_{12} \\
0 & 0 & 0 & 0 \\
0 & 0 & 0 & 0 \\
a_{21} & 0 & 0 & a_{22}
\end{array}\right]
$$

and the map

$$
\Phi_{1}: L_{p}(\mathcal{M}) \otimes M_{2} \rightarrow L_{p}(\mathcal{M}) \otimes M_{4}, a \mapsto \gamma_{1}^{*} a \gamma_{1}
$$

is completely positive. Next, we show that the map

$$
\begin{aligned}
\Phi_{2}: L_{p}(\mathcal{M}) \otimes M_{4} & \rightarrow L_{1}\left(M_{k}\right) \otimes M_{4} \\
& {\left[\begin{array}{ll}
x_{11} & x_{12} \\
x_{21} & x_{22}
\end{array}\right] \mapsto\left[\begin{array}{ll}
S\left(x_{11}\right) & \tilde{T}\left(x_{12}\right) \\
\tilde{T}\left(x_{21}\right) & S\left(x_{22}\right)
\end{array}\right] \text { where } x_{i j} \in L_{p}(\mathcal{M}) \otimes M_{2} }
\end{aligned}
$$

is completely positive. So let $n \in \mathbb{N}$ and $x=\left[x_{i j}\right] \in\left(L_{p}(\mathcal{M}) \otimes M_{4} \otimes M_{n}\right)_{+}$where $x_{i j} \in L_{p}(\mathcal{M}) \otimes M_{4}$. Then $S_{2 n}(x) \pm \tilde{T}_{2 n}(x) \geq 0$, and, by Lemma 2.1

$$
\left[\begin{array}{ll}
S_{2 n}(x) & \tilde{T}_{2 n}(x) \\
\tilde{T}_{2 n}(x) & S_{2 n}(x)
\end{array}\right] \geq 0
$$

We write $x$ as

$$
x=\left[\begin{array}{ll}
x_{i j 11} & x_{i j 12} \\
x_{i j 21} & x_{i j 22}
\end{array}\right]
$$

where $x_{i j l m} \in L_{p}(\mathcal{M}) \otimes M_{2}, i, j \in\{1, \ldots, n\}, l, m=1,2$. Then

$$
\left[\begin{array}{cc}
S_{2 n}(x) & \tilde{T}_{2 n}(x) \\
\tilde{T}_{2 n}(x) & S_{2 n}(x)
\end{array}\right]=\left[\begin{array}{cccc}
S_{n}\left(x_{i j 11}\right) & S_{n}\left(x_{i j 12}\right) & \tilde{T}_{n}\left(x_{i j 11}\right) & \tilde{T}_{n}\left(x_{i j 12}\right) \\
S_{n}\left(x_{i j 21}\right) & S_{n}\left(x_{i j 22}\right) & \tilde{T}_{n}\left(x_{i j 21}\right) & \tilde{T}_{n}\left(x_{i j 22}\right) \\
\tilde{T}_{n}\left(x_{i j 11}\right) & \tilde{T}_{n}\left(x_{i j 12}\right) & S_{n}\left(x_{i j 11}\right) & S_{n}\left(x_{i j 12}\right) \\
\tilde{T}_{n}\left(x_{i j 21}\right) & \tilde{T}_{n}\left(x_{i j 22}\right) & S_{n}\left(x_{i j 21}\right) & S_{n}\left(x_{i j 22}\right)
\end{array}\right] .
$$

We multiply this matrix from left by the scalar matrix $\gamma_{2}=\left[\begin{array}{llll}1 & 0 & 0 & 0 \\ 0 & 0 & 0 & 1\end{array}\right]$ and from right the by its adjoined matrix $\gamma_{2}^{*}$, and get

$$
0 \leq\left[\begin{array}{cc}
S_{n}\left(\left[x_{i j 11}\right]\right) & \tilde{T}_{n}\left(\left[x_{i j 12}\right]\right) \\
\tilde{T}_{n}\left(\left[x_{i j 21}\right]\right) & S_{n}\left(\left[x_{i j 22}\right]\right)
\end{array}\right] .
$$

We multiply the matrix in (4.19) from right by the scalar $2 n \times 2 n$ matrix $\gamma_{3}$ and from left by $\gamma_{3}^{*}$, where $\gamma_{3}$ has 1 at position $(l, m)$ when $(l, m)=(2 i-1, i)$ or $(l, m)=$ $(2 i, n+i)$ for $i \in\{1, \ldots, n\}$ and 0 else. Then the resulting matrix is $\Phi_{2, n}(x)$. This shows that $\Phi_{2}$ is completely positive. Next we define the linear map 


$$
\Phi_{3}: L_{1}\left(M_{k}\right) \otimes M_{4} \rightarrow L_{1}\left(M_{k}\right) \otimes M_{2}, b \mapsto \gamma_{1} b \gamma_{1}^{*}
$$

where $\gamma_{1}$ is the $2 \times 4$ matrix used in the beginning of the proof. Then $\Phi_{3}$ is completely positive. Since $\Phi=\Phi_{3} \circ \Phi_{2} \circ \Phi_{1}$, $\Phi$ is completely positive. This finishes the proof.

For linear maps $S_{1}, S_{2}, T: L_{p}(\mathcal{M}) \rightarrow L_{1}(\mathcal{N})$ we define

$$
\begin{gathered}
{\left[\begin{array}{ll}
S_{1} & T \\
T^{*} & S_{2}
\end{array}\right]: L_{p}(\mathcal{M}) \otimes M_{2} \rightarrow L_{1}(\mathcal{N}) \otimes M_{2}} \\
{\left[\begin{array}{ll}
x_{11} & x_{12} \\
x_{21} & x_{22}
\end{array}\right] \mapsto\left[\begin{array}{ll}
S_{1}\left(x_{11}\right) & T\left(x_{12}\right) \\
T\left(x_{21}^{*}\right)^{*} & S_{2}\left(x_{22}\right)
\end{array}\right]}
\end{gathered}
$$

Lemma 4.15 Let $\mathcal{N}$ be injective, $T: L_{p}(\mathcal{M}) \rightarrow L_{1}(\mathcal{N})$ be completely order bounded, $\quad m, n_{1}, \ldots, n_{m} \in \mathbb{N}, \quad x_{l} \in\left(L_{p}(\mathcal{M}) \otimes M_{2} \otimes M_{n_{l}}\right)_{+}, y_{l} \in\left(\mathcal{N} \otimes M_{2} \otimes\right.$ $\left.M_{n_{l}}\right)_{+}$, for $l=1, \ldots, m$ and $\varepsilon>0$. Then there exist completely positive maps $S_{1}, S_{2}: L_{p}(\mathcal{M}) \rightarrow L_{1}(\mathcal{N})$ such that $\left\|S_{1}\right\|,\left\|S_{2}\right\| \leq\|T\|_{c o b}$ and for the map $\left[\begin{array}{cc}S_{1} & T \\ T^{*} & S_{2}\end{array}\right]$ defined in (4.20) holds

$$
\left\langle\left[\begin{array}{ll}
S_{1} & T \\
T^{*} & S_{2}
\end{array}\right]_{n_{l}}\left(x_{l}\right), y_{l}\right\rangle \geq-\varepsilon, \quad l=1, \ldots m
$$

Proof Let $x_{l}=\left[x_{l, i j}\right], \quad$ where $\quad x_{l, i j} \in L_{p}(\mathcal{M}) \otimes M_{2}, \quad$ and $\quad y_{l}=\left[y_{l, i j}\right]$, where $y_{l, i j} \in \mathcal{N} \otimes M_{2}$, and for $i, j=1, \ldots, n_{l}, l=1, \ldots, m$

$$
x_{l, i j}=\left[\begin{array}{ll}
x_{l, i j 11} & x_{l, i j 12} \\
x_{l, i j 21} & x_{l, i j 22}
\end{array}\right], y_{l, i j}=\left[\begin{array}{ll}
y_{l, i j 11} & y_{l, i j 12} \\
y_{l, i j 21} & y_{l, i j 22}
\end{array}\right] \text {. }
$$

Since $\mathcal{N}$ is injective, there exist by [2], Theorem IV.2.4.4, $k \in \mathbb{N}$ and completely positive contractions $\sigma_{1}: \mathcal{N} \rightarrow M_{k}$ and $\sigma_{2}: M_{k} \rightarrow \mathcal{N}$ such that $\sigma_{1}$ is continuous in the $\sigma$-weak topology and

$$
\begin{aligned}
& \mid \sum_{i, j=1}^{n_{l}}\left(\left\langle T\left(x_{l, i j 21}^{*}\right)^{*}, y_{l, j i 12}-\sigma_{2} \circ \sigma_{1}\left(y_{l, j i 12}\right)\right\rangle\right. \\
& \left.\quad+\left\langle T\left(x_{l, i j 12}\right), y_{l, j i 21}-\sigma_{2} \circ \sigma_{1}\left(y_{l, j i 21}\right)\right\rangle\right) \mid<\varepsilon, l=1, \ldots, m .
\end{aligned}
$$

Since $x_{l}$ and $y_{l}$ are positive, we have $x_{l, i j 21}^{*}=x_{l, j i 12}$ and $y_{l, i j 12}^{*}=y_{l, j i 21}$ for all $l=1, \ldots, m$. Thus, $\left\langle T\left(x_{l, i j 21}^{*}\right)^{*}, y_{l, j i 12}\right\rangle$ and $\left\langle T\left(x_{l, j i 12}\right), y_{l, i j 21}\right\rangle$ are conjugate complex numbers for all $i, j=1, \ldots, n_{l}, l=1, \ldots, m$, and the sum on the left side of this inequality is a real number. Hence the sum is greater than $-\varepsilon$. 
Since $\sigma_{1}$ is continuous in the $\sigma$-weak topology, its adjoint map $\sigma_{1}^{t}$ maps $L_{1}\left(M_{k}\right)$ to $L_{1}(\mathcal{N})$ and is a completely positive contraction. Similarly, the adjoint map $\sigma_{2}^{t}$ maps $L_{1}(\mathcal{N})$ to $L_{1}\left(M_{k}\right)$ and is a completely positive contraction, and $\sigma_{2}^{t} \circ T$ is completely order bounded with $\left\|\sigma_{2}^{t} \circ T\right\|_{c o b} \leq\|T\|_{c o b}$. Now, we apply Proposition 4.14 and get linear maps $S_{1}^{\prime}, S_{2}^{\prime}: L_{p}(\mathcal{M}) \rightarrow L_{1}\left(M_{k}\right)$ such that $\left\|S_{1}^{\prime}\right\|,\left\|S_{2}^{\prime}\right\| \leq\|T\|_{c o b}$ and $\left[\begin{array}{cc}S_{1}^{\prime} & \sigma_{2}^{t} \circ T \\ \sigma_{2}^{t} \circ T^{*} & S_{2}^{\prime}\end{array}\right]$ is completely positive. We put $S_{1}=\sigma_{1}^{t} \circ S_{1}^{\prime}$ and $S_{2}=\sigma_{1}^{t} \circ S_{2}^{\prime}$. Then $\left\|S_{1}\right\|,\left\|S_{2}\right\| \leq\|T\|_{c o b}, S_{1}$ and $S_{2}$ are completely positive, and for all $l=1, \ldots, m$

$$
\begin{aligned}
\left\langle\left[\begin{array}{ll}
S_{1} & T \\
T^{*} & S_{2}
\end{array}\right]_{n_{l}}\left(x_{l}\right), y_{l}\right\rangle=\sum_{i, j=1}^{n_{l}} & \left(\left\langle S_{1}\left(x_{l, i j 11}\right), y_{l, j i 11}\right\rangle\right. \\
& +\left\langle T\left(x_{l, i j 12}\right), y_{l, j i 21}\right\rangle \\
& +\left\langle T\left(x_{l, i j 21}^{*}\right)^{*}, y_{l, j i 12}\right\rangle \\
& \left.+\left\langle S_{2}\left(x_{l, i j 22}\right), y_{l, i j 22}\right\rangle\right) \\
= & \sum_{i, j=1}^{n_{l}}\left(\left\langle\sigma_{1}^{t} \circ S_{1}^{\prime}\left(x_{l, i j 11}\right), y_{l, j i 11}\right\rangle\right. \\
& +\left\langle T\left(x_{l, i j 12}\right), \sigma_{2} \circ \sigma_{1}\left(y_{l, j i 21}\right)\right\rangle \\
& +\left\langle T\left(x_{l, i j 21}^{*}\right)^{*}, \sigma_{2} \circ \sigma_{1}\left(y_{l, j i 12}\right)\right\rangle \\
& \left.+\left\langle\sigma_{1}^{t} \circ S_{2}^{\prime}\left(x_{l, i j 22}\right), y_{l, i j 22}\right\rangle\right) \\
+ & \sum_{i, j=1}^{n_{l}}\left(\left\langle T\left(x_{l, i j 21}^{*}\right)^{*}, y_{l, j i 12}-\sigma_{2} \circ \sigma_{1}\left(y_{l, j i 12}\right)\right\rangle\right. \\
& \left.+\left\langle T\left(x_{l, i j 12}\right), y_{l, j i 21}-\sigma_{2} \circ \sigma_{1}\left(y_{l, j i 21}\right)\right\rangle\right) .
\end{aligned}
$$

Now the first sum of the last expression in (4.22) is equal to

$$
\left\langle\left(\sigma_{1}^{t}\right)_{2 n_{l}} \circ\left[\begin{array}{cc}
S_{1}^{\prime} & \sigma_{2}^{t} \circ T \\
\left(\sigma_{2}^{t} \circ T\right)^{*} & S_{2}^{\prime}
\end{array}\right]_{n_{l}}\left(x_{l}\right), y_{l}\right\rangle,
$$

which is greater than or equal to 0 , because it is a composition of two completely positive maps applied to a positive element. The second sum is greater than $-\varepsilon$ by (4.21). This finishes the proof.

Theorem 4.16 Let $1 \leq p \leq \infty, \mathcal{M}$ and $\mathcal{N}$ be von Neumann algebras, $\mathcal{N}$ injective, and $T: L_{p}(\mathcal{M}) \rightarrow L_{1}(\mathcal{N})$ be a completely order bounded map. Then there exist linear maps $S_{1}, S_{2}: L_{p}(\mathcal{M}) \rightarrow L_{1}(\mathcal{N}),\left\|S_{1}\right\|,\left\|S_{2}\right\| \leq\|T\|_{\text {cob }}$ such that the map 


$$
\begin{gathered}
\Phi: L_{p}(\mathcal{M}) \otimes M_{2} \rightarrow L_{1}(\mathcal{N}) \otimes M_{2} \\
{\left[\begin{array}{ll}
x_{11} & x_{12} \\
x_{21} & x_{22}
\end{array}\right] \mapsto\left[\begin{array}{ll}
S_{1}\left(x_{11}\right) & T\left(x_{12}\right) \\
T\left(x_{21}^{*}\right)^{*} & S_{2}\left(x_{22}\right)
\end{array}\right]}
\end{gathered}
$$

is completely positive.

Proof Let $\mathcal{N}^{*}$ be the dual space of $\mathcal{N}$ and $\mathcal{B}\left(L_{p}(\mathcal{M}), \mathcal{N}^{*}\right)$ be the vector space of all bounded linear maps from $L_{p}(\mathcal{M})$ to $\mathcal{N}^{*}$. By [12], Theorem IV.2.3, $\mathcal{B}\left(L_{p}(\mathcal{M}), \mathcal{N}^{*}\right)$ is isomorphic to the dual space of $L_{p}(\mathcal{M}) \otimes_{\gamma} \mathcal{N}$, where $\|\cdot\|_{\gamma}$ denotes the projective tensor norm. The weak ${ }^{*}$-topology on $\mathcal{B}\left(L_{p}(\mathcal{M}), \mathcal{N}^{*}\right)$ is given by the seminorms $|\langle S(x), y\rangle|, \quad$ where $\quad x \in L_{p}(\mathcal{M}), y \in \mathcal{N}, \quad$ and $\quad S \in \mathcal{B}\left(L_{p}(\mathcal{M}), \mathcal{N}^{*}\right)$. Let $\mathcal{U}=\left\{S \in \mathcal{B}\left(L_{p}(\mathcal{M}), \mathcal{N}^{*}\right) \mid\|S\| \leq\|T\|_{c o b}\right\}$. By the Banach-Alaoglu theorem $\mathcal{U}$ is compact in the weak ${ }^{*}$-topology. Hence $\mathcal{U} \times \mathcal{U}$ is compact in the product weak*topology. For $\quad \varepsilon>0, m \in \mathbb{N}, n_{1}, \ldots, n_{m} \in \mathbb{N}, l=1, \ldots, m, X=\left\{\left(x_{l}, y_{l}\right) \mid x_{l} \in\right.$ $\left.\left(L_{p}(\mathcal{M}) \otimes M_{2} \otimes M_{n_{l}}\right)_{+}, y_{l} \in\left(\mathcal{N} \otimes M_{2} \otimes M_{n_{l}}\right)_{+}\right\}$, let

$$
\begin{aligned}
K(X, \varepsilon)= & \left\{\left(S_{1}, S_{2}\right) \in \mathcal{U} \times \mathcal{U} \mid S_{1}, S_{2}\right. \text { completely positive, } \\
& \left.\left\langle\left[\begin{array}{ll}
S_{1} & T \\
T^{*} & S_{2}
\end{array}\right]_{n_{l}}\left(x_{l}\right), y_{l}\right\rangle \geq-\varepsilon \text { for all }\left(x_{l}, y_{l}\right) \in X\right\} .
\end{aligned}
$$

We will show that $K(X, \varepsilon)$ is closed in the weak*-topology and hence compact. So let $\left(S_{1}, S_{2}\right)$ be in the closure of $K(X, \varepsilon)$. Let $\delta>0$ and $\left(x_{l}, y_{l}\right) \in X$, where

$$
\begin{aligned}
& x_{l}=\left[x_{l, i j}\right], x_{l, i j}=\left[\begin{array}{ll}
x_{l, i j 11} & x_{l, i j 12} \\
x_{l, i j 21} & x_{l, i j 22}
\end{array}\right] \in L_{p}(\mathcal{M}) \otimes M_{2}, \\
& y_{l}=\left[y_{l, i j}\right], \quad y_{l, i j}=\left[\begin{array}{ll}
y_{l, i j 11} & y_{l, i j 12} \\
y_{l, i j 21} & y_{l, i j 22}
\end{array}\right] \in \mathcal{N} \otimes M_{2} .
\end{aligned}
$$

Then we can find $\left(S_{1 \delta}, S_{2 \delta}\right) \in K(X, \varepsilon)$ such that

$$
\begin{aligned}
& \sum_{i, j=1}^{n_{l}}\left|\left\langle S_{1}\left(x_{l, i j 11}\right)-S_{1 \delta}\left(x_{l, i j 11}\right), y_{l, j i 11}\right\rangle\right|<\delta \\
& \sum_{i, j=1}^{n_{l}}\left|\left\langle S_{2}\left(x_{l, i j 22}\right)-S_{2 \delta}\left(x_{l, i j 22}\right), y_{l, j i 22}\right\rangle\right|<\delta,
\end{aligned}
$$

and therefore 


$$
\begin{aligned}
& \left|\left\langle\left[\begin{array}{cc}
S_{1} & T \\
T^{*} & S_{2}
\end{array}\right]_{n_{l}}\left(x_{l}\right), y_{l}\right\rangle-\left\langle\left[\begin{array}{cc}
S_{1 \delta} & T \\
T^{*} & S_{2 \delta}
\end{array}\right]_{n_{l}}\left(x_{l}\right), y_{l}\right\rangle\right| \\
= & \mid \sum_{i, j=1}^{n_{l}}\left\langle S_{1}\left(x_{l, i j 11}\right)-S_{1 \delta}\left(x_{l, i j 11}\right), y_{l, j i 11}\right\rangle \\
& +\sum_{i, j=1}^{n_{l}}\left\langle S_{2}\left(x_{l, i j 22}\right)-S_{2 \delta}\left(x_{l, i j 22}\right), y_{l, j i 22}\right\rangle \mid<2 \delta
\end{aligned}
$$

Now $\left\langle\left[\begin{array}{cc}S_{1 \delta} & T \\ T^{*} & S_{2 \delta}\end{array}\right]_{n_{l}}\left(x_{l}\right), y_{l}\right\rangle$ is a positive number. Hence the imaginary part

$$
\left|\operatorname{Im}\left\langle\left[\begin{array}{ll}
S_{1} & T \\
T^{*} & S_{2}
\end{array}\right]_{n_{l}}\left(x_{l}\right), y_{l}\right\rangle\right|<2 \delta
$$

and the real part

$$
\operatorname{Re}\left\langle\left[\begin{array}{ll}
S_{1} & T \\
T^{*} & S_{2}
\end{array}\right]_{n_{l}}\left(x_{l}\right), y_{l}\right\rangle>\left\langle\left[\begin{array}{cc}
S_{1 \delta} & T \\
T^{*} & S_{2 \delta}
\end{array}\right]_{n_{l}}\left(x_{l}\right), y_{l}\right\rangle-2 \delta \geq \varepsilon-2 \delta
$$

Since $\delta$ is arbitrary, $\left\langle\left[\begin{array}{cc}S_{1} & T \\ T^{*} & S_{2}\end{array}\right]_{n_{l}}\left(x_{l}\right), y_{l}\right\rangle$ is a real number which is greater than or equal to $-\varepsilon$. Similarly, we show that $\left\|S_{1}\right\|,\left\|S_{2}\right\| \leq\|T\|_{c o b}$ and $S_{1}, S_{2}$ are completely positive. So $\left(S_{1}, S_{2}\right) \in K(X, \varepsilon)$.

The sets $K(X, \varepsilon), m \in \mathbb{N}, X=\left\{\left(x_{1}, y_{1}\right), \ldots\left(x_{m}, y_{m}\right)\right\}, \varepsilon>0$ have the finite intersection property. If we have sets $K\left(X_{l}, \varepsilon_{l}\right), l=1, \ldots, m$, we put $X=\bigcup_{l=1}^{m} X_{l}$ and $\varepsilon=\min \left\{\varepsilon_{1}, \ldots, \varepsilon_{m}\right\}$. Then $K(x, \varepsilon) \subseteq \cap_{l=1}^{m} K\left(X_{l}, \varepsilon_{l}\right)$. Combining the finite intersection property and the compactness in the weak*-topology, we get

$$
\bigcap_{X, \varepsilon} K(X, \varepsilon) \neq \emptyset
$$

Any pair $\left(S_{1}, S_{2}\right)$ in this intersection has the property that $\left[\begin{array}{cc}S_{1} & T \\ T^{*} & S_{2}\end{array}\right]$ is completely positive and $\left\|S_{1}\right\|,\left\|S_{2}\right\| \leq\|T\|_{c o b}$. Since there is a projection $z \in \mathcal{N}^{* *}$ which works as a projection from $\mathcal{N}^{*}$ to $L_{1}(\mathcal{N})$, the maps $S_{1}^{\prime}=z S_{1}$ and $S_{2}^{\prime}=z S_{2}$ have the desired properties.

We close this section with an example of a completely order bounded map which is not decomposable when $q>2 p$. The boundaries for $p$ and $q$ are not sharp. The goal is to show that there are many combinations of $p$ and $q$ for which completely order bounded does not imply decomposable. Since we are working with semifinite von Neumann algebras in the example, we use the $L_{p}$-spaces associated with the trace, because they are easier to handle than the Haagerup-Terp construction. For 
$m \in \mathbb{N}$, let $l_{q}^{m}$ be the vector space $\mathbb{C}^{m}$ equipped with norm $\|c\|_{q}=\left(\sum_{i=1}^{m}\left|c_{i}\right|^{q}\right)^{1 / q}$ where $c=\left(c_{1}, \ldots, c_{m}\right)$. Let

$$
e_{1}, \ldots, e_{m} \text { be the standard base of } l_{q}^{m} \text {. }
$$

Before we show our example, we need some formulas to estimate the completely order bounded norm and the decomposable norm for linear maps from $L_{p}(\mathcal{M})$ to $l_{q}^{m}$.

Lemma $\quad 4.17$ Let $1 \leq p<\infty, 1 \leq q<\infty, \frac{1}{p}+\frac{1}{p^{\prime}}=1, m \in \mathbb{N}, g_{1}, \ldots, g_{m} \in L_{p^{\prime}}(\mathcal{M})$ and

$$
T: L_{p}(\mathcal{M}) \rightarrow l_{q}^{m}, \quad f \mapsto \sum_{k=1}^{m}\left\langle f, g_{k}\right\rangle e_{k}
$$

Then

$$
\|T\|_{c o b} \leq \sup \left\{\left(\sum_{k=1}^{m}\left\|a g_{k} b\right\|_{1}^{q}\right)^{1 / q} \mid a, b \in L_{2 p}(\mathcal{M}),\|a\|_{2 p},\|b\|_{2 p} \leq 1\right\} .
$$

Proof Let $n \in \mathbb{N}$ and $x \in L_{p}(\mathcal{M}) \otimes M_{n},\|x\|_{p, n} \leq 1$. By Theorem 3.4 and Theorem 2.3, there exist $a, b \in L_{2 p}(\mathcal{M}),\|a\|_{2 p},\|b\|_{2 p} \leq 1, y \in \mathcal{M} \otimes M_{n},\|y\|_{\infty} \leq 1$ such that $x=\left(b \otimes 1_{n}\right) y\left(a \otimes 1_{n}\right)$. Let $\varepsilon_{i j}$ be as in (3.2) and $y=\sum_{i, j=1}^{n} y_{i j} \otimes \varepsilon_{i j}$. Then we have

$$
T_{n}(x)=\sum_{k=1}^{m} \sum_{i, j=1}^{n}\left\langle a g_{k} b, y_{i j}\right\rangle e_{k} \otimes \varepsilon_{i j}
$$

For $k=1, \ldots, m$ let $\varphi_{k}: \mathcal{M} \rightarrow \mathbb{C}, f \mapsto\left\langle a g_{k} b, f\right\rangle$. Then $\varphi_{k}$ is completely order bounded and $\left\|\varphi_{k}\right\|_{c o b}=\left\|\varphi_{k}\right\|=\left\|a g_{k} b\right\|_{1}$. Since $\|y\|_{\infty} \leq 1$, we have

$$
0 \leq\left[\begin{array}{cc}
\left\|\varphi_{k}\right\| 1_{n} & \left(\varphi_{k}\right)_{n}(y) \\
\left(\varphi_{k}\right)_{n}(y)^{*} & \left\|\varphi_{k}\right\| 1_{n}
\end{array}\right]
$$

Then we get

$$
\begin{aligned}
0 & \leq \sum_{k=1}^{m} e_{k} \otimes\left[\begin{array}{cc}
\left\|\varphi_{k}\right\| 1_{n} & \left(\varphi_{k}\right)_{n}(y) \\
\left(\varphi_{k}\right)_{n}(y)^{*} & \left\|\varphi_{k}\right\| 1_{n}
\end{array}\right] \\
& =\left[\begin{array}{cc}
\sum_{k=1}^{m}\left\|\varphi_{k}\right\| e_{k} \otimes 1_{n} & T_{n}(x) \\
T_{n}(x)^{*} & \sum_{k=1}^{m}\left\|\varphi_{k}\right\| e_{k} \otimes 1_{n}
\end{array}\right]
\end{aligned}
$$

Hence 


$$
\left\|T_{n}(x)\right\|_{q, n} \leq\left\|\sum_{k=1}^{m}\right\| a g_{k} b\left\|_{1} e_{k}\right\|_{q}=\left(\sum_{k=1}^{m}\left\|a g_{k} b\right\|_{1}^{q}\right)^{\frac{1}{q}}
$$

which proves (4.24).

Lemma 4.18 Let $2 \leq q<\infty, \alpha \in M_{n}$, and $\varepsilon_{i i}$ be as in (3.2) for $i=1, \ldots, n$. Then

$$
\sum_{i=1}^{n}\left\|\alpha \varepsilon_{i i}\right\|_{q}^{q} \leq\|\alpha\|_{q}^{q}
$$

Proof Let $\alpha^{*} \alpha=\beta=\left[\beta_{i j}\right]_{i, j=1}^{n}$. Let $\left|\alpha \varepsilon_{i i}\right|$ be the positive matrix of the polar decomposition of $\alpha \varepsilon_{i i}$. Then $\left|\alpha \varepsilon_{i i}\right|^{2}=\varepsilon_{i i} \beta \varepsilon_{i i}=\beta_{i i} \varepsilon_{i i}$ which implies $\left|\alpha \varepsilon_{i i}\right|^{q}=\beta_{i i}^{q / 2} \varepsilon_{i i}$ and

$$
\sum_{i=1}^{n}\left\|\alpha \varepsilon_{i i}\right\|_{q}^{q}=\sum_{i=1}^{n} \operatorname{Tr}\left(\beta_{i i}^{q / 2} \varepsilon_{i i}\right)=\sum_{i=1}^{n} \beta_{i i}^{q / 2} .
$$

Since $\beta$ is positive, it has eigenvalues $\lambda_{1}, \ldots, \lambda_{n} \geq 0$, and there is a unitary matrix $u=\left[u_{i j}\right] \in M_{n}$ such that $\beta=u^{*} \operatorname{diag}\left(\lambda_{1}, \ldots, \lambda_{n}\right) u$. Especially, we have $\beta_{i i}=\sum_{l=1}^{n}\left|u_{l i}\right|^{2} \lambda_{l}$. From $u$ being unitary, it follows that $\sum_{i=1}^{n}\left|u_{l i}\right|^{2}=1$ for $l=1, \ldots, n$. Since $q \geq 2$, the function $\phi:[0, \infty) \rightarrow \mathbb{R}, t \mapsto t^{q / 2}$ is convex. Hence we get

$$
\begin{aligned}
\sum_{i=1}^{n} \beta_{i i}^{q / 2} & =\sum_{i=1}^{n} \phi\left(\sum_{l=1}^{n} \lambda_{l}\left|u_{l i}\right|^{2}\right) \leq \sum_{i=1}^{n} \sum_{l=1}^{n}\left|u_{l i}\right|^{2} \phi\left(\lambda_{l}\right) \\
& =\sum_{l=1}^{n} \lambda_{l}^{q / 2} \sum_{i=1}^{n}\left|u_{l i}\right|^{2}=\sum_{l=1}^{n} \lambda_{l}^{q / 2}=\operatorname{Tr}\left(\beta^{q / 2}\right)=\|\alpha\|_{q}^{q}
\end{aligned}
$$

For $n \in \mathbb{N}$ let $\varepsilon_{i j}$ be as in (3.2) and $e_{i}$ as in (4.23). Then we define the linear map

$$
T: L_{p}\left(M_{n}\right) \rightarrow l_{n}^{q}, \quad T(x)=\sum_{i=1}^{n} \operatorname{Tr}\left(\left(\varepsilon_{1 i}+\varepsilon_{i 1}\right) x\right) e_{i}
$$

Proposition 4.19 Let $1 \leq p<\infty, 2 p<q<\infty$, and $T$ as in (4.25). Then $\|T\|_{c o b} \leq 2$.

Proof Let $\varepsilon>0$. By Lemma 4.17, there exist $a, b \in L_{2 p}\left(M_{n}\right),\|a\|_{2 p},\|b\|_{2 p} \leq 1$ such that

$$
\|T\|_{c o b} \leq\left(\sum_{i=1}^{n}\left\|a\left(\varepsilon_{i 1}+\varepsilon_{1 i}\right) b\right\|_{1}^{q}\right)^{1 / q}+\varepsilon .
$$

Since for all $i=1, \ldots, n$ we have $\left\|a\left(\varepsilon_{i 1}+\varepsilon_{1 i}\right) b\right\|_{1} \leq\left\|a \varepsilon_{i 1} b\right\|_{1}+\left\|a \varepsilon_{1 i} b\right\|_{1}$ we get 


$$
\left(\sum_{i=1}^{n}\left\|a\left(\varepsilon_{i 1}+\varepsilon_{1 i}\right) b\right\|_{1}^{q}\right)^{1 / q} \leq\left(\sum_{i=1}^{n}\left\|a \varepsilon_{i 1} b\right\|_{1}^{q}\right)^{1 / q}+\left(\sum_{i=1}^{n}\left\|a \varepsilon_{1 i} b\right\|_{1}^{q}\right)^{1 / q}
$$

Let $\frac{1}{q}+\frac{1}{q^{\prime}}=1$. Then $\left\|a \varepsilon_{i 1} b\right\|_{1}=\left\|a \varepsilon_{i i} \varepsilon_{i 1} b\right\|_{1} \leq\left\|a \varepsilon_{i i}\right\|_{q} \cdot\left\|\varepsilon_{i 1} b\right\|_{q^{\prime}}$. Since $q>2 p \geq 2$ we have $q^{\prime}<2 \leq 2 p$. Hence there exists a real number $s>1$ such that $\frac{1}{q^{\prime}}=\frac{1}{2 p}+\frac{1}{s}$. By the generalized Hölder's inequality, we have $\left\|\varepsilon_{i 1} b\right\|_{q^{\prime}} \leq\left\|\varepsilon_{i 1}\right\|\left\|_{s}\right\| b \|_{2 p} \leq 1$. We apply Lemma 4.18 and get

$$
\sum_{i=1}^{n}\left\|a \varepsilon_{i 1} b\right\|_{1}^{q} \leq \sum_{i=1}^{n}\left\|a \varepsilon_{i i}\right\|_{1}^{q} \leq\|a\|_{q}^{q}
$$

Since $q>2 p \geq 2$, we have $\|a\|_{q} \leq\|a\|_{2 p} \leq 1$. Hence

$$
\left(\sum_{i=1}^{n}\left\|a \varepsilon_{i 1} b\right\|_{1}^{q}\right)^{1 / q} \leq 1
$$

Similarly, we get

$$
\left(\sum_{i=1}^{n}\left\|a \varepsilon_{1 i} b\right\|_{1}^{q}\right)^{1 / q} \leq 1
$$

Since $\varepsilon$ was arbitrary, this finishes the proof.

Proposition 4.20 Let $1 \leq p<\infty, 1 \leq q<\infty$, and $T$ as in (4.25). Then $\|T\|_{\text {dec }} \geq n^{1 / 2 q}$.

Proof Since $T$ is self-adjoint, we can apply [1], Lemma 2.18 and 2.19, and get

$$
\|T\|_{d e c}=\inf \left\{\|S\| \mid S: L_{p}\left(M_{n}\right) \rightarrow l_{q}^{n}, S \pm T \text { is completely positive }\right\} .
$$

In [1], the case $p=q$ is considered only. But the proof also works for $p \neq q$. Let $S: L_{p}\left(M_{n}\right) \rightarrow l_{q}^{n}$ be a linear map such that $S \pm T$ is completely positive. There exist $b_{1}, \ldots, b_{n} \in M_{n+}$ such that $S(f)=\sum_{k=1}^{n}\left\langle b_{k}, f\right\rangle e_{k}$ for all $f \in L_{p}\left(M_{n}\right)$. Since $S \pm T$ are positive, we have

$$
\sum_{k=1}^{n}\left\langle b_{k} \pm\left(\varepsilon_{1 k}+\varepsilon_{k 1}\right), f\right\rangle e_{k} \geq 0 \text { for all } f \in L_{p}\left(M_{n}\right)_{+} .
$$

This means that $b_{k} \pm\left(\varepsilon_{1 k}+\varepsilon_{k 1}\right) \geq 0$ for $k=1, \ldots, n$. Let $b_{k}=\left[b_{k, i j}\right]$. For $k=1$ we have $b_{1}-2 \varepsilon_{11} \geq 0$ which implies $b_{1,11} \geq 2$. For $k>1$ we have 


$$
\begin{aligned}
0 & \leq\left[\begin{array}{cc}
e_{k}^{t} & 0 \\
0 & e_{1}^{t}
\end{array}\right]\left[\begin{array}{cc}
b_{k} & \varepsilon_{1 k}+\varepsilon_{k 1} \\
\varepsilon_{1 k}+\varepsilon_{k 1} & b_{k}
\end{array}\right]\left[\begin{array}{cc}
e_{k} & 0 \\
0 & e_{1}
\end{array}\right] \\
& =\left[\begin{array}{cc}
e_{k}^{t} b_{k} e_{k} & e_{k}^{t} \varepsilon_{k 1} e_{1} \\
e_{1}^{t} \varepsilon_{1 k} e_{k} & e_{1}^{t} b_{k} e_{1}
\end{array}\right]=\left[\begin{array}{cc}
b_{k, k k} & 1 \\
1 & b_{k, 11}
\end{array}\right]
\end{aligned}
$$

We compute the determinant of the last matrix in (4.26) and get $b_{k, k} b_{k, 11} \geq 1$. Since the diagonal elements of $b_{k}$ are positive, we get $b_{k, k k} \geq \frac{1}{b_{k, 11}}$ for all $k \geq 2$. For $k=$ $1, \ldots, n$ we have

$$
\left\|S\left(\varepsilon_{k k}\right)\right\|_{q}^{q}=\left\|\sum_{j=1}^{n}\left\langle b_{j}, \varepsilon_{k k}\right\rangle e_{j}\right\|_{q}^{q}=\left\|\sum_{j=1}^{n} b_{j, k k} e_{j}\right\|_{q}^{q}=\sum_{j=1}^{n}\left(b_{j, k k}\right)^{q} .
$$

For $k=1$ we have $\left\|S\left(\varepsilon_{11}\right)\right\|_{q}^{q}=\sum_{j=1}^{n}\left(b_{j, 11}\right)^{q}$. For $k>1$ we have $\left\|S\left(\varepsilon_{k k}\right)\right\|_{q}^{q} \geq\left(b_{k, k k}\right)^{q} \geq \frac{1}{\left(b_{k, 11}\right)^{q}}$. Hence

$$
\|S\|^{q} \geq \max \left\{\sum_{j=1}^{n}\left(b_{j, 11}\right)^{q}, \frac{1}{\left(b_{k, 11}\right)^{q}}, k=2, \ldots, n\right\} .
$$

If there is some $k, 2 \leq k \leq n$ such that $b_{k, 11} \leq n^{-1 / 2 q}$, then

$$
\|S\|^{q} \geq \frac{1}{\left(b_{k, 11}\right)^{q}} \geq \sqrt{n} .
$$

If $b_{k, 11} \geq n^{-1 / 2 q}$ for all $k \geq 2$, we have

$$
\begin{aligned}
\|S\|^{q} & \geq \sum_{k=1}^{n}\left(b_{k, 11}\right)^{q} \geq 2^{q}+(n-1) n^{-1 / 2} \\
& =\frac{2^{q} \sqrt{n}+n-1}{\sqrt{n}} \\
& \geq \sqrt{n}
\end{aligned}
$$

Combining (4.27) and (4.28), we get

$$
\|S\| \geq n^{\frac{1}{2 q}} .
$$

Since $S$ was arbitrary with $S \pm T$ completely positive, this finishes the proof.

Now we can show our counterexample. Let $\mathcal{M}=\oplus_{k=1}^{\infty} M_{k}$. On $\mathcal{M}$ we have the semifinite, normal, faithful trace $\tau_{1}(x)=\sum_{k=1}^{\infty} \operatorname{Tr}_{k}\left(x_{k}\right)$ where $x=\oplus_{k=1}^{\infty} x_{k}$ and $\operatorname{Tr}_{k}$ is the usual trace on $M_{k}$. For any projection $e \in \mathcal{M}$, we have $\tau_{1}(e) \geq 1$ and therefore every $\tau_{1}$-measurable operator affiliated with $\mathcal{M}$ is a bounded operator. Hence for all $1 \leq p<\infty$, we can write $L_{p}(\mathcal{M})=\left\{x=\oplus_{k=1}^{\infty} x_{k} \mid \sum_{k=1}^{\infty}\left\|x_{k}\right\|_{p}^{p}<\infty\right\}$ with norm $\|x\|_{p}=\left(\sum_{k=1}^{\infty}\left\|x_{k}\right\|_{p}^{p}\right)^{1 / p} . \quad$ Let $\quad \mathcal{N}=\oplus_{k=1}^{\infty} l_{\infty}^{k} . \quad$ Then $\quad L_{q}(\mathcal{N})=\left\{f=\oplus_{k=1}^{\infty} f_{k} \mid\right.$ 
$\left.\sum_{k=1}^{\infty}\left\|f_{k}\right\|_{q}^{q}<\infty\right\}$ with norm $\|f\|_{q}=\left(\sum_{k=1}^{\infty}\left\|f_{k}\right\|_{q}^{q}\right)^{1 / q}$. For $1 \leq p, q<\infty, q>2 p$ let

$$
T: L_{p}(\mathcal{M}) \rightarrow L_{q}(\mathcal{N}), \quad T\left(\oplus_{k=1}^{\infty} x_{k}\right)=\oplus_{k=1}^{\infty} T_{k}\left(x_{k}\right)
$$

where $T_{k}$ is defined as in (4.25). We claim that $T$ is completely order bounded and $\|T\|_{c o b} \leq 2$. To show this, let $n \in \mathbb{N}$ and $x \in L_{p}\left(\mathcal{M} \otimes M_{n}\right),\|x\|_{p, n} \leq 1$. According to Theorem 3.4, there exist $f, g \in L_{P}(\mathcal{M})_{+}$such that

$$
\|f\|_{p}=\|g\|_{p}=\|x\|_{p, n} \text { and }\left[\begin{array}{cc}
f \otimes 1_{n} & x \\
x^{*} & g \otimes 1_{n}
\end{array}\right] \geq 0 .
$$

Then we have $x=\oplus_{k=1}^{\infty} x_{k}, x_{k} \in L_{p}\left(M_{k}\right) \otimes M_{n}, f=\oplus_{k=1}^{\infty} f_{k}, f_{k} \in L_{p}\left(M_{k}\right)_{+}$and $g=$ $\oplus_{k=1}^{\infty} g_{k}, g_{k} \in L_{p}\left(M_{k}\right)_{+}$with

$$
\left[\begin{array}{cc}
f_{k} \otimes 1_{n} & x_{k} \\
x_{k}^{*} & g_{k} \otimes 1_{n}
\end{array}\right] \geq 0 \text { for all } k \in \mathbb{N} .
$$

By Proposition 4.19, we have $\left\|T_{k}\right\|_{c o b} \leq 2$ for all $k \in \mathbb{N}$. By definition of completely order boundedness, for all $k \in \mathbb{N}$, there exist $h_{1, k}, h_{2, k} \in\left(l_{k}^{q}\right)_{+}$, such that

$$
\left[\begin{array}{cc}
h_{1, k} \otimes 1_{n} & T_{k, n}\left(x_{k}\right) \\
T_{k, n}\left(x_{k}\right)^{*} & h_{2, k} \otimes 1_{n}
\end{array}\right] \geq 0 \text { and }\left\|h_{1, k}\right\|_{q},\left\|h_{2, k}\right\|_{q} \leq \frac{1}{2}\left\|T_{k}\right\|_{c o b}\left(\left\|f_{k}\right\|_{p}+\left\|g_{k}\right\|_{p}\right) .
$$

We put $h_{1}=\oplus_{k=1}^{\infty} h_{1, k}$ and $h_{2}=\oplus_{k=1}^{\infty} h_{2, k}$. Then $\left\|h_{1}\right\|_{q},\left\|h_{2}\right\|_{q} \leq 2$ and

$$
\left[\begin{array}{cc}
h_{1} \otimes 1_{n} & T_{n}(x) \\
T_{n}(x)^{*} & h_{2} \otimes 1_{n}
\end{array}\right] \geq 0
$$

which shows that $\|T\|_{c o b} \leq 2$.

Next, suppose that $T$ is decomposable. Then there exists a completely positive map $S: L_{p}(\mathcal{M}) \rightarrow L_{q}(\mathcal{N})$, such that $S \pm T$ are completely positive. For every $j \in \mathbb{N}$, the embedding $I_{j}: L_{p}\left(M_{j}\right) \rightarrow L_{p}(\mathcal{M}), x \mapsto(\ldots, 0, x, 0, \ldots)$ and the projection $P_{j}: L_{q}(\mathcal{N}) \rightarrow l_{q}^{j}, \oplus_{k=1}^{\infty} y_{k} \mapsto y_{j}$ are completely positive and have norm less than or equal to 1 . We have $T_{j}=P_{j} \circ T \circ I_{j}$ and put $S_{j}=P_{j} \circ S \circ I_{j}$. Then $S_{j} \pm T_{j}$ are completely positive. We apply Proposition 4.20 and get

$$
\infty>\|S\| \geq\left\|S_{j}\right\| \geq\left\|T_{j}\right\|_{d e c} \geq j^{1 / 2 q} \text { for all } j \in \mathbb{N}
$$

which gives a contradiction. Thus $T$ is not decomposable.

Acknowledgements The author thanks the anonymous reviewers for pointing out simplifications of some proofs and typing errors.

Funding Open Access funding enabled and organized by Projekt DEAL.

Open Access This article is licensed under a Creative Commons Attribution 4.0 International License, which permits use, sharing, adaptation, distribution and reproduction in any medium or format, as long as you give appropriate credit to the original author(s) and the source, provide a link to the Creative Commons licence, and indicate if changes were made. The images or other third 
party material in this article are included in the article's Creative Commons licence, unless indicated otherwise in a credit line to the material. If material is not included in the article's Creative Commons licence and your intended use is not permitted by statutory regulation or exceeds the permitted use, you will need to obtain permission directly from the copyright holder. To view a copy of this licence, visit http://creativecommons.org/licenses/by/4.0/.

\section{References}

1. Arhancet, C., Kriegler, C.: Projections, multipliers and decomposable maps on noncommutative $L^{p}$ spaces, arXiv:1707.05591v14 (2018)

2. Blackadar, B.: Operator Algebras: Theory of $\mathrm{C}^{*}$-Algebras and von Neumann Algebras. Springer, New York (2006)

3. Effros, E., Ruan, Z.-J.: Operator Spaces. Oxford University Press, Oxford (2000)

4. Haagerup, U.: Decomposition of completely bounded maps on operator algebras, Unpublished manuscript (1980)

5. Haagerup, U.: Injectivity and decomposition of completely bounded maps, pp. 170-222, In: Operator Algebras and their connection with Topology and Ergodic Theory. Lecture Notes in Mathematics 1132, Springer (1985)

6. Junge, M., Ruan, Z.-J.: Decomposable maps on non-commutative $L_{p}$-spaces, 355-381, In: Operator algebras, quantization, and noncommutative geometry. Contemporary Mathematics 365, Journal of the American Mathematical Society, Providence, RI (2004)

7. Junge, M., Ruan, Z.-J., Xu, Q.: Rigid $\mathcal{O} \mathcal{L}$ s structures of non-commutative $L_{p}$-spaces associated with hyperfinite von Neumann algebras. Math. Scand. 95, 63-95 (2005)

8. Paulsen, V.: Completely bounded maps On $C^{*}$-algebras and invariant operator ranges. Proc. Am. Math. Soc. 86, 91-96 (1982)

9. Pisier, G.: Regular operators between non-commutative $L_{p}$-spaces. Bull. Sci. Math. 119, 95-118 (1995)

10. Pisier, G.: Introduction to operator space theroy. Cambridge University Press, Cambridge (2003)

11. Schmitt, L.M.: The Radon-Nikodym theorem for $L^{p}$-spaces of $W^{*}$-algebras. Publ. RIMS Kyoto Univ. 22, 1025-1034 (1986)

12. Takesaki, M.: Theory of operator algebras I and II. In: Encyclopaedia of Mathematical Sciences, vol. 124 and 125. Springer (2002 and 2003)

13. Terp, M.: $L^{p}$ spaces associated with von Neumann algebras. Copenhagen Univercity, Copenhagen (1981)

14. Wittstock, G.: Ein operatorwertiger Hahn-Banach Satz. J. Funct. Anal. 40, 127-150 (1981) 